\title{
Pseudoholomorphic maps into folded symplectic four-manifolds
}

\author{
JENS VON BERGMANN
}

\begin{abstract}
Every oriented 4-manifold admits a stable folded symplectic structure, which in turn determines a homotopy class of compatible almost complex structures that are discontinuous across the folding hypersurface ("fold") in a controlled fashion. We define folded holomorphic maps, ie pseudoholomorphic maps that are discontinuous across the fold. The boundary values on the fold are mediated by tunneling maps which are punctured $\mathcal{H}$-holomorphic maps into the folding hypersurface with prescribed asymptotics on closed characteristics.
\end{abstract}

Our main result is that the linearized operator of this boundary value problem is Fredholm, under the simplifying assumption that we have circle-invariant folds.

As examples we characterize the moduli space of maps into the folded elliptic fibration $E^{F}(1)$ and we construct examples of degree $d$ rational maps into $S^{4}$. Moreover we explicitly give the moduli space of degree 1 rational maps into $S^{4}$ and show that it possesses a natural compactification.

This aims to generalize the tools of holomorphic maps to all oriented 4-manifolds by utilizing folded symplectic structures rather than other types of pre-symplectic structures as initiated by Taubes in $[24 ; 25 ; 27 ; 26]$.

32Q65, 58J32; 53C15, 57R17

\section{Introduction}

In his seminal 1985 paper [11], M Gromov showed how to extend tools of complex geometry to the symplectic category. In the last decade this has led to a vibrant new field based on the study of "pseudoholomorphic curves" in symplectic manifolds, yielding many powerful results regarding invariants and the recognition of symplectic manifolds.

Unfortunately, these methods do not apply to all manifolds - many manifolds do not admit symplectic forms. There are different possibilities to extend the theory of pseudoholomorphic curves to a broader class of manifolds. One approach, being pursued by $\mathrm{C}$ Taubes, begins with the observation that every compact oriented 4manifold with intersection form that is not negative definite admits a closed 2-form 
that degenerates along a disjoint union of circles. Taubes has made a detailed study of the behavior of pseudoholomorphic curves approaching these circles $[24 ; 25 ; 27 ; 26]$.

In [6] A Cannas da Silva, V Guillemin and C Woodward introduced the notion of folded symplectic structures, which we describe in Section 1.1. Every orientable 4-manifold admits a folded symplectic structure [5], and by results in Baykur [2] we may further assume that the fold is stable (see Definition 1.4).

In this article we set up a boundary value problem for closed oriented folded symplectic 4-manifolds with circle-invariant folds.

The 4 -sphere $S^{4}$ with its canonical folded symplectic structure is of this form. We compute the moduli space of folded holomorphic maps of degree 1 into $S^{4}$ and give examples of higher degree maps.

Folded holomorphic maps are discontinuous across the fold, a feature that is in stark contrast to properties of pseudoholomorphic maps. We explain why this is natural and also necessary in order to obtain a well-defined moduli space. The discontinuity is controlled by tunneling maps which are the central objects of this theory. Intuitively, folded holomorphic maps can be thought of "smoothings" of pseudoholomorphic maps into each component of the complement of the folding hypersurface equipped with degenerate structures as in Symplectic field theory [9] or in Ionel and Parker [16].

\subsection{Folded symplectic manifolds}

We begin by recalling some definitions and basic properties of folded symplectic manifolds.

Definition 1.1 (Folded symplectic structure) Let $X$ be a smooth $2 n$-dimensional manifold. A folded symplectic structure $\omega$ is a closed 2-form such that $\omega^{n}$ is transverse to 0 (so $Z=\left(\omega^{n}\right)^{-1}(0)$ is empty or a smooth codimension 1 hypersurface) and $\left.\omega^{n-1}\right|_{Z}$ is nonvanishing. $Z$ is called the fold.

The last condition means that the kernel $E=\operatorname{ker}(\omega)$ of $\omega$, which by transversality is a real 2-plane bundle over $Z$, is transverse to $T Z$. This is equivalent to the requirement that

$$
L=\operatorname{ker}\left(\left.\omega\right|_{Z}\right) \subset T Z
$$

is a 1 -dimensional foliation. $L$ is called the characteristic foliation. Thus intrinsically, the fold of a folded symplectic manifold is indistinguishable from an orientable hypersurface in a symplectic manifold.

Folded symplectic structures, like symplectic and contact structures, have a standard local model. 
Theorem 1.2 For every folded symplectic form $\omega$ on a $2 n$-dimensional manifold, there exist local coordinates near the fold such that $\omega$ has the form

$$
x_{1} d x_{1} \wedge d x_{2}+d x_{3} \wedge d x_{4}+\ldots+d x_{2 n-1} \wedge d x_{2 n},
$$

where the fold is locally given by $\left\{x_{1}=0\right\}$.

More generally, in [6] it is proved that for any $\alpha \in \Omega^{1}(Z)$ that does not vanish on $L$, we can extend the inclusion $i: Z \hookrightarrow X$ of the fold to an orientation-preserving diffeomorphism

$$
\phi:(-\varepsilon, \varepsilon) \times Z \rightarrow U
$$

onto a tubular neighborhood $U$ of the fold such that

$$
\phi^{*} \omega=\pi^{*} i^{*} \omega+\frac{1}{2} d\left(r^{2} \pi^{*} \alpha\right)
$$

where $r$ is the coordinate function on $(-\varepsilon, \varepsilon)$ and $\pi$ is the projection $\pi(r, z)=z$. By possibly rescaling the volume form we assume for simplicity that $r=\operatorname{det}(\omega)$ on $U$.

Definition 1.3 A morphism $\psi: X_{1} \rightarrow X_{2}$ of folded symplectic manifolds $\left(X_{1}, \omega_{1}\right)$ and $\left(X_{2}, \omega_{2}\right)$ is a diffeomorphism satisfying

$$
\psi^{*} \omega_{2}=\omega_{1} .
$$

Such morphisms automatically take folds to folds.

A Cannas da Silva showed in [5] using an h-principle that every oriented 4-manifold admits a folded symplectic structure. To define folded holomorphic maps we require a special structure along the fold. I Baykur used folded Lefschetz pencils in [2] to show that every oriented 4-manifold admits a "nicely folded Kähler structure". In particular this implies that we may assume the fold is of contact type; Equation (1.2) can be refined to

$$
\phi^{*} \omega=d\left(\left(\frac{1}{2} r^{2}+1\right) \pi^{*} \alpha\right) .
$$

Thus the following Definition, which allows for more general folds than contact type, does not pose a serious restriction. We borrow from Hofer and Zehnder [14], Bourgeois, Eliashberg, Hofer, Wysocki and Zehnder [4] and Eliashberg, Kim and Polterovich [10].

Definition 1.4 A folded symplectic manifold $(X, \omega)$ together with a choice of 1-form $\alpha$ on the fold $Z$ is called stable if

$$
\alpha \wedge \omega>0 \quad \text { and } \quad \operatorname{ker}\left(\left.\omega\right|_{Z}\right) \subset \operatorname{ker}(d \alpha) .
$$


Such a choice of 1 -form $\alpha$ defines an almost contact form $(\omega, \alpha)$ on $Z$. In particular $\alpha$ defines a section $R$ of the line bundle $L$ by the normalizing condition

$$
\alpha(R)=1 .
$$

The flow generated by $R$ induces an $\mathbb{R}$-action on $Z$ preserving $\omega$ and $\alpha$. The kernel of $\alpha$ gives a symplectic subbundle $\left(F=\operatorname{ker}(\alpha),\left.\omega\right|_{F}\right)$ over $Z$ such that

$$
T_{Z} X=E \oplus F
$$

A parametrized closed characteristic is an injective map $x: S^{1} \rightarrow Z$ satisfying

$$
d x\left(\partial_{\theta}\right)=T \cdot R, \text { for some } T \in(0, \infty) .
$$

A closed characteristic is an image of a parametrized closed characteristic and we let $\mathcal{R}$ be the set of closed characteristics equipped with the disjoint union topology. For each closed characteristic $x \in \mathcal{R}$, the corresponding constant $T=T_{x}$ for a parametrized closed characteristic representing it is called the minimal period. In the case of circle-invariant folds (see Definition 1.6) we have that $T_{x}=1$ for all $x \in \mathcal{R}$.

Now choose a background metric $g^{\prime}$ on $X$. To simplify future computations we assume that in the Darboux chart (1.2),

$$
\left.\phi^{*} g^{\prime}\right|_{U}=d r \otimes d r+\alpha \otimes \alpha+g_{F}
$$

where $g_{F}$ is a metric on $F$ that is compatible with $\left.\omega\right|_{Z}$. Mimicking the standard procedure to generate a compatible triple on a symplectic manifold using the background metric $g^{\prime}$, we obtain a folded triple $(\omega, g, J)$ on $X \backslash Z$ satisfying the compatibility conditions

$$
\begin{aligned}
J^{*} \omega & =\omega \\
g(u, v) & =\omega(u, J v) .
\end{aligned}
$$

Near the fold the triple has the following "standard" form.

Lemma 1.5 The complex structure $\left.J\right|_{X_{ \pm} \backslash Z}$ has one-sided limits $J_{ \pm}$on $T_{Z} X$ such that the splitting (1.5) is $J_{ \pm}$-invariant, $\left.J_{+}\right|_{F}=\left.J_{-}\right|_{F}$ and $\left.J_{+}\right|_{E}=-\left.J_{-}\right|_{E}$.

Moreover, $E$ and $F$ extend to a transverse pair of $J$-invariant subbundles of $T X$ over a neighborhood $U \subset X$ of $Z$, also denoted by $E$ and $F$, such that

$$
\omega_{E}=\operatorname{det}(\omega) \mu, \quad g_{E}=|\operatorname{det}(\omega)| h, \quad J_{E}=\operatorname{sign}(\operatorname{det}(\omega)) \tilde{J}
$$

where $(\mu, h, \widetilde{J})$ is a smooth compatible triple on $E$ with $\widetilde{J} \partial_{r}=R$ on $Z$. 
Proof Define the $g^{\prime}$-skew-endomorphism $A$ of $T X$ by $\omega(u, v)=g^{\prime}(A u, v)$. This exists and is unique since $g^{\prime}$ is positive definite. Observe that over $Z,\left.A\right|_{E}=0$ and $\left.A\right|_{F}$ is nondegenerate. Since $Z$ is compact there exists $\delta>0$ with $|\lambda|>\delta$ for all eigenvalues of $\left.A\right|_{F}$. Thus there exists a neighborhood $U$ of $Z$ such that $\left|\lambda_{1}\right|<\delta / 2<|\lambda|$ over $U$, where $\pm \lambda_{1}$ are the smallest eigenvalues of $A$ and $\pm \lambda$ are the second smallest eigenvalues of $A$. Define $E$ to be the 2-plane bundle over $U$ given by the real eigenspace spanned by $\pm \lambda_{1}$ and set $F=E^{\perp_{g^{\prime}}}$. Then for $u \in E$ and $v \in F$ we have $A u=\tilde{u} \in E$ and

$$
\omega(u, v)=g^{\prime}(A u, v)=g^{\prime}(\widetilde{u}, v)=0 .
$$

Therefore $\omega$ splits as $\omega_{E} \oplus \omega_{F}$. $A$ also splits as $A=A_{E} \oplus A_{F}$ because the real eigenspaces corresponding to different pairs of complex conjugate eigenvalues of a skew-endomorphism are orthogonal. Since $A_{F}$ is nondegenerate, the usual polarization procedure produces a canonical compatible triple there.

Recall that we assumed $r=\operatorname{det}(\omega)$ on a neighborhood $U$ of $Z$, where $r$ is the coordinate function from Equation (1.1). Since $\omega$ is folded symplectic, $\omega_{E}=r \cdot \mu$ for some nondegenerate positive 2-form $\mu$ on $E$. Therefore $A_{E}=r \cdot \widetilde{A}_{E}$, where $\tilde{A}_{E}=g_{E}^{-1} \mu$ is a nondegenerate skew-endomorphism of $E$. By polarization of $\tilde{A}_{E}$ we obtain a smooth compatible triple $(\mu, h, \widetilde{J})$ on $E$, and using $A_{E}$ instead we get a compatible triple $\left(\omega_{E}, g_{E}, J_{E}\right)$ on $E \backslash E_{Z}$ satisfying Equation (1.6).

By Equation (1.2), $\left.\mu\right|_{E}=d r \wedge \alpha$ on $Z$ and thus $\tilde{A}_{E}=R \otimes d r-\partial_{r} \otimes \alpha=\widetilde{J}$.

The complex structure $\widetilde{J}$ allows us to define a complement $K$ of $L$ in $E$ by $K=\widetilde{J} L$, so we can refine the splitting (1.5) over $Z$ to

$$
T_{Z} X=K \oplus L \oplus F .
$$

Equations (1.6) show that $J$ is discontinuous across the fold in the $E$ directions. However, on $U$ we may define two smooth complex structures, denoted by $J^{ \pm}$, such that $\left.J^{ \pm}\right|_{X_{ \pm}}=J$ by choosing $J_{E}^{ \pm}= \pm \widetilde{J}$.

We define folded holomorphic maps for all stable folded symplectic 4-manifolds, but at this stage most proofs rely on the following additional structure that we assume along the fold.

Definition 1.6 A stable folded symplectic manifold has a circle-invariant fold (or $S^{1}$-invariant fold) if the flow of the associated characteristic vector field defines a free $S^{1}$-action on $Z$. 
Folded symplectic manifolds with $S^{1}$-invariant folds are especially easy to work with. They also occur frequently. The standard folded symplectic structure on the spheres (described in the next section) is of this type. Connected sums of symplectic 4-manifolds always have folded symplectic structures of this type [6]. More generally, connected sum along symplectomorphic symplectic submanifolds of arbitrary codimension with symplectomorphic normal bundles in symplectic 4-manifolds produces folded symplectic structures with $S^{1}$-invariant folds. This can be seen by carefully mimicking the symplectic connect sum construction in McCarthy and Wolfson [19] or Ionel and Parker [17].

$S^{1}$-invariant folds have a special structure, as described in Lemma 1.7 below. We will use this lemma repeatedly in later sections. Whenever we work with $S^{1}$-invariant folds we assume that the background metric $g^{\prime}$ was chosen to be $S^{1}$-invariant on the fold, resulting in an $S^{1}$-invariant compatible triple there.

Lemma 1.7 In the case of an $S^{1}$-invariant fold, $Z$ is an $S^{1}=\mathbb{R} / \mathbb{Z}$ bundle over a symplectic manifold $\left(V, \omega_{V}\right)$ with projection $\pi_{V}: Z \rightarrow V$ such that

(1) $\omega_{Z}=\pi_{V}^{*} \omega_{V}$

(2) there exists an $\omega_{V}$ compatible almost complex structure $J_{V}$ on $V$ such that $\left.d \pi_{V}\right|_{F}$ is $\left(J, J_{V}\right)$ linear.

Moreover, the 1-form $\alpha$ may be chosen such that

$$
d \alpha=C \cdot \omega_{Z}, \quad C=c_{1}(Z) / \operatorname{vol}(V)
$$

where $c_{1}$ is the first Chern class of the circle bundle $Z \rightarrow V$ and $\operatorname{vol}(V)$ is taken with respect to $\omega_{V}$.

Proof Since the $S^{1}$-action on $Z$ is free we can exhibit $Z$ as an $S^{1}$ bundle

$$
\pi_{V}: Z \rightarrow V
$$

over a closed $(2 n-2)-$ dimensional manifold $V$. The kernel of $\omega$ coincides with the vertical subspace and $\mathcal{L}_{R} \omega=0$, so $\omega$ is $S^{1}$-invariant. Thus there exists a 2 -form $\omega_{V}$ on $V$ such that $\omega=\pi_{V}^{*} \omega_{V}$. One readily checks that $\omega_{V}$ is nondegenerate and closed. Because $\operatorname{ker}\left(d \pi_{V}\right)$ is transverse to $F$,

$$
\left.d \pi_{V}\right|_{F}(z): F \rightarrow T_{\pi_{V}(z)} S
$$

is an isomorphism for each $z \in Z$. Since the complex structure $J$ on $F$ is invariant under the $S^{1}$-action, this map induces a complex structure $J_{V}$ on $V$ so that $\left.d \pi_{V}\right|_{F}$ is $\left(J, J_{V}\right)$ linear. 
To see Equation (1.8) let $\alpha_{0}$ be a connection 1 -form on $Z$, ie $\alpha_{0}$ is invariant under the $S^{1}$-action and satisfies $\alpha_{0}(R)=1$. Then $\iota_{R} d \alpha_{0}=\mathcal{L}_{R} \alpha_{0}=0$, so $d \alpha_{0}=\pi_{V}^{*} \omega_{0}$ is the pullback of a 2 -form $\omega_{0}$ on $V$ which is just the curvature of the connection $\alpha_{0}$ and therefore represents $c_{1}(Z)$.

Since $\omega_{V}$ is a volume form on $V$ there exists a constant $c \in \mathbb{R}$ with

$$
\int_{V}\left(c \omega_{V}-\omega_{0}\right)=0
$$

so $\left(c \omega_{V}-\widetilde{\omega}\right)=d \beta$ for some 1 -form $\beta$ on $V$. With the gauge transformation $\alpha=\alpha_{0}+\pi_{V}^{*} \beta$ we still have $\alpha(R)=1$, and

$$
d \alpha=d \alpha_{0}+\pi_{V}^{*} d \beta=\pi_{V}^{*} \omega_{0}+\pi_{V}^{*}\left(c \omega_{V}-\omega_{0}\right)=c \pi_{V}^{*} \omega_{V}=c \omega_{Z} .
$$

Henceforth we assume that all folded symplectic manifolds in question are compact, connected, 4-dimensional and have stable folds. If we additionally assume the folds to be circle-invariant, we assume that the 1 -form $\alpha$ is chosen to satisfy Equation (1.8).

\section{Motivating example}

To motivate what follows we will investigate possibilities to define pseudoholomorphic maps into $S^{4}$ with canonical folded symplectic structure as defined below. One overruling principle is that we want to obtain well-behaved moduli spaces of such maps. More precisely we are looking for a notion of pseudoholomorphic maps into folded symplectic manifolds such that the linearized equations at a solution give rise to a Fredholm operator and that the solutions are stable under perturbations of the structures involved.

Recall that $S^{4}$ does not admit any symplectic form nor a continuous almost complex structure. Nor does $S^{4}$ admit the pre-symplectic structure used by Taubes. Therefore the answer to the question how to generalize pseudoholomorphic maps to this setting is far from obvious. We let ourselves be guided by the folded symplectic structure $\omega$. Since $\omega$ is nondegenerate on $X \backslash Z$, the usual procedure to construct a compatible triple will yield an almost complex structure $J$ there. Then it is clear what a pseudoholomorphic map from a Riemann surface $(\Sigma, j)$ into $X \backslash Z$ is, namely a map with $(j, J)$-linear differential. Since the fold $Z$ separates $X$ into $X_{ \pm}$this means that maps from a connected domain into $X \backslash Z$ will have image in only one side $X_{+}$or $X_{-}$of the fold. The question then is how to allow maps to "cross the fold", ie have image on both sides of the fold. 
One way to do this is to choose an almost complex structure on $X \backslash Z$ that degenerates along $Z$ in a way that $X_{ \pm}$has "cylindrical ends" in the sense of Symplectic field theory [9]. This then reduces to the problem of holomorphic curves relative to closed characteristics as discussed in [16], [9] and [4]. In effect this is treating the two sides $X_{ \pm}$as separate manifolds with boundary.

We try and find the analogue of holomorphic curves with nondegenerate almost complex structure for folded symplectic manifold, while being guided by the hope that these will limit to the relative curves discussed above as we degenerate the almost complex structure. To do this we define a circle-invariant folded symplectic structure on $S^{4}$ together with a compatible almost complex structure as in Lemma 1.5.

View $S^{4}$ as the unit sphere in $\mathbb{R}^{5}$. Then we have

- the restriction of the coordinate projection $\Pi: \mathbb{R}^{5}=\mathbb{R} \times \mathbb{R}^{4} \rightarrow \mathbb{R}^{4}$ to $S^{4}$, and

- the stereographic projections $\sigma_{ \pm}: S^{4} \backslash( \pm 1,0,0,0,0) \rightarrow \mathbb{R}^{4}$.

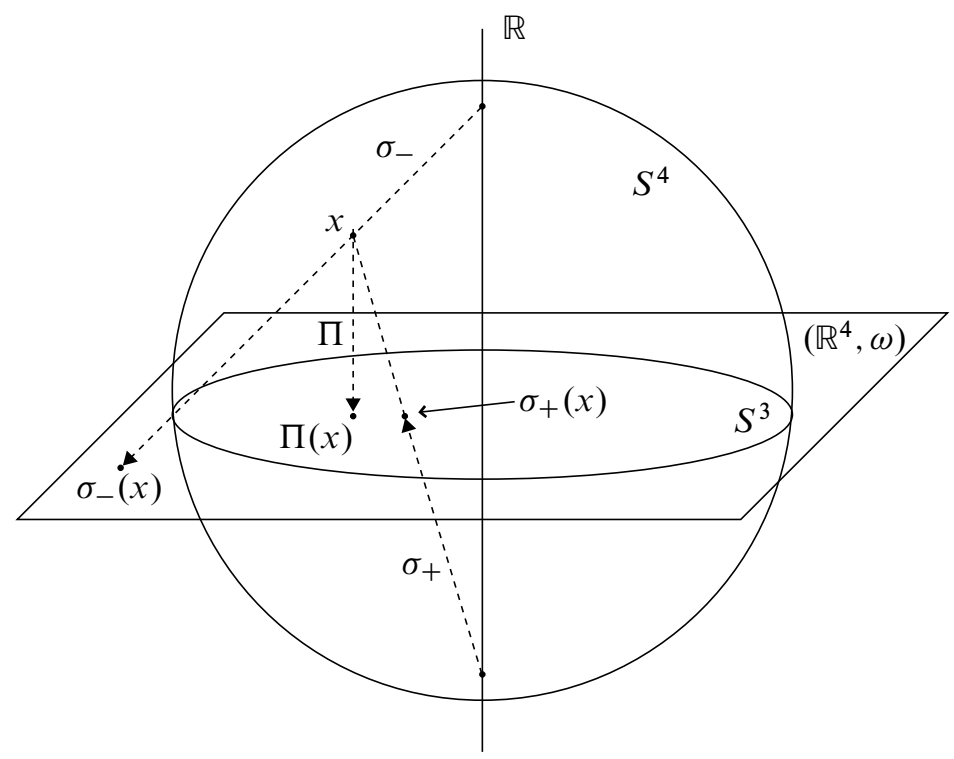

Figure 1: Folded structures on $S^{4}$

Let $\omega_{0}$ and $J_{0}$ be the standard symplectic and complex structures on $\mathbb{R}^{4}$. Then $\omega=\Pi^{*}\left(\frac{1}{\pi} \omega_{0}\right)$ is a folded symplectic form. The orientation induced by the folded symplectic form agrees (disagrees) with the canonical orientation on $S^{4}$ on the upper (lower) hemisphere $S_{+}^{4}\left(S_{-}^{4}\right)$, the fold $Z=S^{3}$ is the intersection of $S^{4}$ with the equatorial plane $\left\{\left(x_{0}, x_{1}, x_{2}, x_{3}, x_{4}\right) \mid x_{0}=0\right\}$. Choose the 1 -form $\alpha$ on $Z=S^{3}$ 
to be the canonical contact structure on $S^{3}$, ie $\alpha$ is the restriction of the canonical 1 -form

$$
\alpha=\frac{1}{2 \pi}\left(x_{1} d x_{2}-x_{2} d x_{1}+x_{3} d x_{4}-x_{4} d x_{3}\right)
$$

to $S^{3}$. Thus $d \alpha=\omega_{Z}$.

With the almost complex structure $J$ defined as $\left.J\right|_{S_{ \pm}^{4}}=\sigma_{ \pm}^{*} J_{0}$ and the symmetric bilinear form $g(u, v)=\omega(u, J v)$ we obtain a folded compatible triple $(J, \omega, g)$ on $S^{4}$. Note that all structures are invariant under the $S^{1}=\mathbb{R} / \mathbb{Z}$-action on $Z$ given by the characteristic flow, ie $t \cdot(z, w)=\left(e^{2 \pi i t} z, e^{2 \pi i t} w\right)$.

The map

$$
\tau: S^{4} \rightarrow S^{4}, \quad\left(x_{0}, x_{1}, x_{2}, x_{3}, x_{4}\right) \mapsto\left(-x_{0}, x_{1}, x_{2}, x_{3}, x_{4}\right)
$$

is a biholomorphic involution on $S^{4}$ exchanging the upper and lower hemisphere and fixing the fold.

The bundle $F=\operatorname{ker}(\alpha)$ is given by the contact planes of the fold $S^{3}$, and $E$ is spanned by the characteristic direction given by the vertical subspaces of the Hopf fibration and the "radial" direction.

Any nontrivial $J$-holomorphic map from a Riemann surface $(\Sigma, j)$ has to cross the fold, since each side is biholomorphic to $B^{4} \subset \mathbb{C}^{2}$ which is contractible and therefore does not admit nontrivial holomorphic curves by their energy minimizing property. Suppose for simplicity that the preimage of the fold is a closed 1-dimensional separating submanifold $\sigma$ on the domain $\Sigma$, cutting it into two parts $\Sigma_{+}$mapping into $S_{+}^{4}$ and $\Sigma_{-}$mapping into $S_{-}^{4}$.

Investigating the orientations on the transverse bundle $E$ near the fold reveals that it is impossible to have holomorphic maps of class $C^{1}$ (or even $C^{0}$ ) into $S^{4}$ that cross the fold (see also Remark 3.12). To remedy this one may look at curves that are holomorphic on $\Sigma_{+}$, antiholomorphic on $\Sigma_{-}$and continuous across the fold. Here we face the problem that too many curves exists. For example, any holomorphic map from the upper hemisphere of $S^{2}$ into $S_{+}^{4}$ with boundary on the fold can be completed by an antiholomorphic map from the lower hemisphere into $S_{-}^{4}$ simply by reflection through the equator on domain and target, ie using the antiholomorphic involution of the hemispheres of $S^{2}$ and the holomorphic involution $\tau$ on $S^{4}$. Thus there exist an infinite-dimensional space of solutions in this case.

The reason for this is revealed by studying this as a boundary value problem. The condition of "continuous images" does not give rise to elliptic boundary conditions and therefore cannot lead to a Fredholm problem. 
One possible remedy is to impose additional constraints to cut down the solutions to a finite-dimensional space. But there is no evident way of doing this so that the structure of the solution space is stable under perturbations and that allows for sufficient interaction between the two sides of the fold.

Another approach is to allow discontinuous images. We think of this as holomorphic curves that leave the fold at a location that is different from where they enter, the relation between these is given by a tunneling map in the fold. This way we define a Fredholm problem for discontinuous pseudoholomorphic maps into folded symplectic manifolds. Roughly speaking, a folded holomorphic map consists of

- a domain $(\Sigma, j)$ with 1 -dimensional submanifold $\sigma$ separating $\Sigma$ into $\Sigma_{+}$and $\Sigma_{-}$

- $J$-holomorphic maps $u_{+}:\left(\Sigma_{+}, \sigma\right) \rightarrow\left(X_{+}, Z\right)$ and $u_{-}:\left(\Sigma_{-}, \sigma\right) \rightarrow\left(X_{-}, Z\right)$

- a pair of tunneling maps in $Z$ connecting $u_{+}(\sigma)$ to a closed characteristic and then continuing on to connect to $u_{-}(\sigma)$.

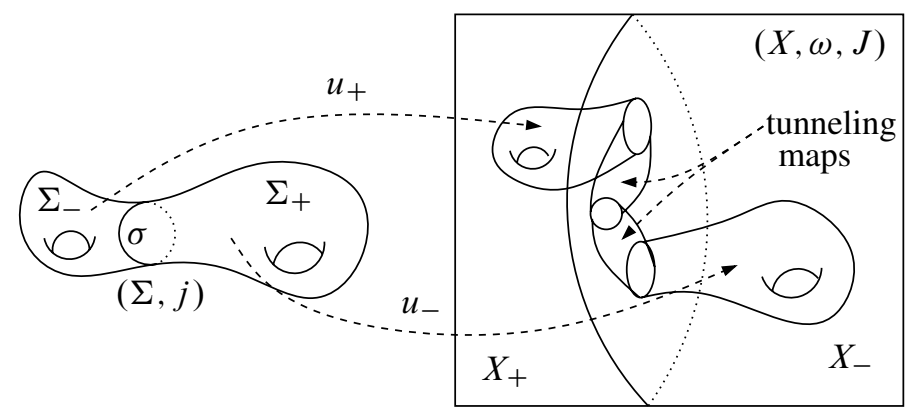

Figure 2: The map tunnels through the fold, exiting the fold at a location that is different from where it entered.

We will make this precise in the following sections. In order to convince the reader that it is natural to consider discontinuous maps in the context of folded symplectic manifolds we will first give a trivial example.

Consider the complex elliptic fibration $X=E(1)$ and let $T \hookrightarrow E(1)$ be a regular fiber. Perturb the almost complex structure $J$ so that a neighborhood $X_{-}$of $T$ is biholomorphic to $X_{-}=D \times T$ with a product complex structure, where $D$ is the closed unit disk in $\mathbb{C}$ with the canonical complex structure and $T=\{0\} \times T$ with its original complex structure. Set $X_{+}=E(1) \backslash X_{-}$.

After choosing $z_{0} \in S^{1}$ we define for $z \in S^{1}$ the element $\bar{z} \in S^{1}$ by reflection through $z_{0}$, ie $z \cdot \bar{z}=z_{0}$. Using the two self-maps of the boundary $Z=\partial X_{ \pm}=S^{1} \times T$

$$
\iota(z, w)=(z, w) \quad \text { and } \quad \iota_{F}(z, w)=(\bar{z}, w),
$$


we construct the manifolds

$$
E(1)=X_{+} \sqcup_{\iota} X_{-} \quad \text { and } \quad E^{F}(1)=X_{+} \sqcup_{\iota_{F}} \bar{X}_{-}
$$

with almost complex structure inherited from each piece. With a little more care we can ensure that $E^{F}(1)$ comes equipped with a folded symplectic structure, independent of the choice of $z_{0}$.

Consider the biholomorphism

$$
\Psi: E(1) \backslash Z \rightarrow E^{F}(1) \backslash Z
$$

given by the identity map on each piece, $X_{+}$and $X_{-}$.

This setup suggest the following definition for folded holomorphic maps into the folded symplectic manifolds $E^{F}(1)$. Let $\sigma$ be a separating submanifold in a Riemann surface $\Sigma$, separating $\Sigma$ into two pieces $\Sigma_{+}$and $\Sigma_{-}$.

Definition 2.1 A folded holomorphic map consists of a pair of maps $\left(u_{+}, u_{-}\right)$such that

$$
u_{ \pm}: \Sigma_{ \pm} \rightarrow X_{ \pm} \quad \text { and } \quad u_{ \pm}=\left.\Psi \circ u\right|_{\Sigma_{ \pm}}
$$

for some $J$-holomorphic $u: \Sigma \rightarrow E(1)$.

Note that there is an $S^{1}$ ambiguity in the gluing map $\iota_{F}$, so there is really an $S^{1}$ family of these folded holomorphic maps. It is clear from the definition that this will yield a well-defined moduli space, although the maps are necessarily discontinuous.

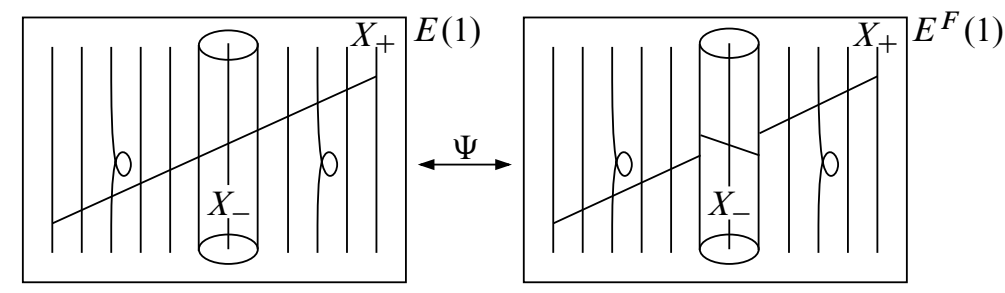

Figure 3: Folded holomorphic maps in $E^{F}(1)$

In the following we will show how to define folded holomorphic maps into stable folded symplectic manifolds that reduce to Definition 2.1 in the case of $E^{F}(1)$.

Note that the pieces $u_{+}$and $u_{-}$of a folded holomorphic map into $E^{F}(1)$ have boundary values in

$$
\Delta^{Z}=\left\{\left(\left.u_{+}\right|_{\sigma},\left.u_{-}\right|_{\sigma}\right)\right\}=\operatorname{graph}(\Psi) \in \operatorname{Map}(\sigma, Z) \times \operatorname{Map}(\sigma, Z)
$$


We call $\Delta^{Z}$ the folded diagonal. The above definitions work in the case where the fold $Z$ has the structure of a trivial $S^{1}$-bundle with the characteristic foliation being vertical. To define folded holomorphic maps into more general folded symplectic manifolds we need to generalize the folded diagonal so that it continues to give elliptic boundary conditions for the maps $u_{+}$and $u_{-}$.

\section{Folded holomorphic maps}

We define folded holomorphic maps and lay the functional analytic foundation for the later sections. We start by describing the domains of folded holomorphic maps, then we set up the Sobolev spaces and lastly we set up the PDE.

\subsection{Folded domains}

We define the domains of folded holomorphic maps. Due to the additional structure needed for the tunneling maps this is more involved than one might at first expect.

Definition 3.1 (Folded domain) A smooth folded domain $\mathcal{D}$ consists of

(i) closed Riemann surfaces $\left(\Sigma_{0}, j_{0}, \mathbf{p}_{0}\right)$ and $\left(\Sigma_{1}, j_{1},\{p\}\right)$ of genus $g_{0}$ and $g_{1}$ with $\mathbf{p}_{0}=\left\{p_{1}, \ldots p_{n}\right\} \subset \Sigma_{0}$

(ii) continuous functions $\tau_{i}: \Sigma_{i} \rightarrow \mathbb{R}$ so that with

$$
\Sigma_{i}^{+}=\tau_{i}^{-1}[0, \infty) \quad \text { and } \quad \Sigma_{i}^{-}=\tau_{i}^{-1}(-\infty, 0]
$$

their restrictions $\tau_{i}^{ \pm}=\left.\tau_{i}\right|_{\Sigma_{i}^{ \pm}}$extend to smooth functions on $\Sigma_{i}$ with zeros of at most finite order along $\sigma_{i}=\tau_{i}^{-1}(0)=\left(\tau_{i}^{ \pm}\right)^{-1}(0)$ with matching order of vanishing at each point of $\sigma_{i}, i=0,1$

(iii) $p \in \Sigma_{1}^{-}$

(iv) a function $g: \Sigma_{0}^{+} \rightarrow \mathbb{R}$ and a diffeomorphism $\psi: \Sigma_{0}^{+} \rightarrow \Sigma_{1}^{+}$satisfying the conditions

$$
\psi^{*} j_{1}=j_{0} \quad \text { and } \quad \psi^{*} \tau_{1}=e^{g} \tau_{0} .
$$

We set $\mathbf{p}_{0}^{ \pm}=\mathbf{p}_{0} \cap \Sigma_{0}^{ \pm}$to be the marked points contained in $\Sigma_{0}^{ \pm}$. The zero sets $\sigma_{0}$ and $\sigma_{1}$ are called the domain folds. The map $\psi$ gives an identification of $\sigma_{0}$ and $\sigma_{1}$, so we will refer to the domain folds simply by $\sigma$. To simplify notation we set

$$
\Sigma_{ \pm}=\Sigma_{0}^{ \pm}, \quad S=\Sigma_{1}^{-}, \quad \dot{S}=S \backslash\{p\} .
$$




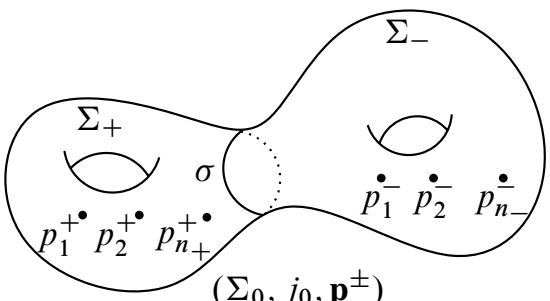

$\left(\Sigma_{0}, j_{0}, \mathbf{p}^{ \pm}\right)$

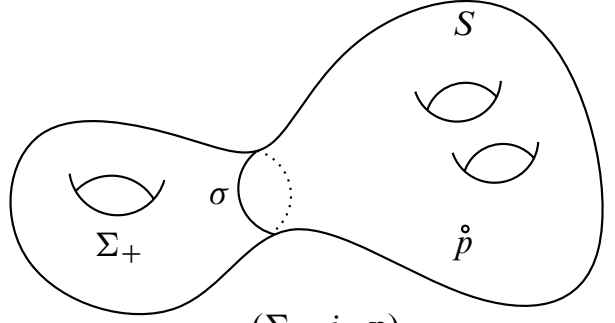

$\left(\Sigma_{1}, j_{1}, \mathbf{p}\right)$

Figure 4: Folded domains

When no confusion can occur we will drop the subscripts on $j_{i}$ and $\tau_{i}$.

The purpose of the functions $\tau_{0}$ and $\tau_{1}$ is to give possible locations of the domain fold $\sigma$, but we are not interested at the specific values of $\tau_{i}$ away from their zero sets.

Definition 3.2 The group

$$
\mathcal{G}=\operatorname{Map}\left(\Sigma_{1}, \mathbb{R}\right) \times \operatorname{Diff}^{+}\left(\Sigma_{0}, \mathbf{p}_{0}\right) \times \operatorname{Diff}^{+}\left(\Sigma_{1},\{p\}\right)
$$

is called the gauge group.

Lemma 3.3 The gauge group $\mathcal{G}$ acts on the space of folded domains in the following way. Given $\left(f, \phi_{0}, \phi_{1}\right) \in \mathcal{G}$ the folded domain transforms according to

$$
\begin{array}{rlrl}
j_{i} & \mapsto \phi_{i}^{*} j_{i}=d \phi_{i}^{-1} \circ j_{i} \circ d \phi_{i}, \\
\tau_{0} & \mapsto \tau_{0} \circ \phi_{0}, & \tau_{1} & \mapsto e^{f} \tau_{1} \circ \phi_{1}, \\
\mathbf{p}_{0} & \mapsto \phi_{0}^{*}\left(\mathbf{p}_{0}\right), \quad p \mapsto \phi_{1}^{*}(p), \\
\psi & \mapsto \phi_{1}^{-1} \circ \psi \circ \phi_{0}, & \\
g & \mapsto g \circ \phi_{0}+\left(\phi_{1}^{-1} \circ \psi \circ \phi_{0}\right)^{*} f .
\end{array}
$$

Remark 3.4 (1) The sets $\mathbf{p}_{0}^{ \pm}$are disjoint unless some of the marked points lie on $\sigma_{0}$.

(2) Let be $\hat{S}$ the oriented blowup of $\dot{S}$ as in [4, Section 4.3], ie $\hat{S}$ is a compact surface with boundary where $\widehat{S} \backslash \dot{S}$ consists of the circle $\left(T_{p} S \backslash 0\right) / \mathbb{R}^{>0}$. Here $\mathbb{R}^{>0}=(0, \infty)$.

We define the associated 2-dimensional $C^{0}$-cycles with boundary $\widetilde{\Sigma}_{ \pm}$by gluing $\Sigma_{+}$to $\hat{S}$ to get $\widetilde{\Sigma}_{+}=\widehat{\Sigma}_{1}$ or by gluing $\Sigma_{-}$to $-\widehat{S}$ to get $\widetilde{\Sigma}_{-}$, where the gluing is done along $\sigma . \widetilde{\Sigma}_{+}$is a smooth Riemann surface, whereas the same only holds for $\widetilde{\Sigma}_{-}$in the case that the domain fold $\sigma$ is a manifold. 
(3) Since the order of vanishing of $\tau^{ \pm}=\left.\tau_{0}\right|_{\Sigma_{ \pm}}$at each point of $\sigma$ is the same we may define the "gap-function"

$$
a: \sigma \rightarrow(0, \infty)
$$

at a point $z \in \sigma$ by choosing a path $\gamma:[-1,1] \rightarrow \Sigma$ with $\gamma([0,1]) \in \Sigma_{+}$, $\gamma([-1,0]) \in \Sigma_{-}$and $\gamma(0)=z$ and setting

$$
a(z)=\lim _{t \rightarrow 0} \frac{\tau_{-} \circ \gamma(|t|)}{\tau_{+} \circ \gamma(-|t|)} .
$$

Note that this is independent of the choice of path $\gamma$. At a generic point $z \in \sigma$ where $\tau_{ \pm}(z)$ vanish to first order we have

$$
a(z)=\frac{d \tau_{-}(z)}{d \tau_{+}(z)},
$$

where the quotient of forms is to be understood as being taken after evaluating on an arbitrary normal vector to $\sigma$. This is well defined since $d \tau_{ \pm}$are collinear at $z$ as they vanish along the direction of $\sigma$.

\subsection{Sobolev spaces of maps}

To proceed we need to give a precise definition of the Sobolev spaces we use. We work with the nondegenerate metric $\widetilde{g}$ that, with the notation of Lemma 1.5, leaves the splitting $T U=E \oplus F$-invariant, equals $g$ on $F$ and $\mu$ on $E$ in a neighborhood $U^{\prime} \subset \bar{U}^{\prime} \subset U$ of the fold and equals $g$ outside of $U$ so that $J_{ \pm}$is antisymmetric with respect to $\widetilde{g}$. In particular

$$
\tilde{g}=d r \otimes d r+\alpha \otimes \alpha+g_{F} \quad \text { and } \quad g_{Z}=\left.\tilde{g}\right|_{Z}=\alpha \otimes \alpha+g_{F}
$$

on $U^{\prime}$ and $Z$, respectively.

Consider a folded domain $\mathcal{D}$ as in Definition 3.1. We follow the definitions from Booß-Bavnbek and Wojciechowski [3] for Sobolev spaces on manifolds with boundary.

Definition 3.5 Fix a Riemannian metric in the conformal class of $j$ on $\Sigma_{0}$ and positive integers $k, p$ with $k p>2$. Let $U_{ \pm} \subset \Sigma_{0}$ be open subsets properly containing $\Sigma_{ \pm}$and that are of the same homotopy type as $\Sigma_{ \pm}$. Then let $W^{k, p}\left(\Sigma_{ \pm}, X_{ \pm}\right)$be the Banach manifold consisting of maps $f: \Sigma_{ \pm} \rightarrow X_{ \pm}$that are restrictions of maps $\tilde{f}: U_{ \pm} \rightarrow X$ of class $W^{k, p}$ to $\Sigma_{ \pm}$that send the domain fold $\sigma$ into $Z$. 
$W^{k, p}\left(\Sigma_{ \pm}, X_{ \pm}\right)$is a smooth separable Banach manifold modeled locally at a map $u_{ \pm} \in W^{k, p}\left(\Sigma_{ \pm}, X_{ \pm}\right)$on the space

$$
\left\{\zeta \in W^{k, p}\left(u_{ \pm}^{*} T X\right)|\zeta|_{\sigma} \in W^{k-\frac{1}{p}, p}\left(\left.u_{ \pm}\right|_{\sigma} ^{*} T Z\right)\right\} .
$$

Next we define the Banach manifolds of maps from punctured surfaces into $Z$. We follow largely the traditional treatment found in Schwarz [22], Hofer, Wysocki and Zehnder [13] or Dragnev [7], except that we avoid using the auxiliary $\mathbb{R}$-factor in the "symplectization" as it does not add any information.

For a closed Riemann surface $\Sigma$ with finitely many punctures $\left\{p_{k}\right\}$ and $\dot{\Sigma}=\Sigma \backslash\left\{p_{k}\right\}$ we let $W_{\text {loc }}^{k, p}(\dot{\Sigma}, Z)$ be the space of maps from $\dot{\Sigma}$ to $Z$ that, in local coordinates, are in $W_{\mathrm{loc}}^{k, p}\left(\mathbb{R}^{2}, \mathbb{R}^{3}\right)$.

For a Riemann surface $\Sigma$ with boundary and finitely many punctures we assume that $\Sigma \subset \Sigma^{\prime}$ for some open Riemann surface $\Sigma^{\prime}$ of the same homotopy type and we let $W_{\text {loc }}^{k, p}(\dot{\Sigma}, Z)$ be the space of maps from $\dot{\Sigma}$ to $Z$ that are restrictions of maps in $W_{\mathrm{loc}}^{k, p}\left(\dot{\Sigma}^{\prime}, Z\right)$

Let $\left(\Sigma, j,\left\{p_{k}\right\}\right)$ be a Riemann surface (possibly with boundary) with conformal structure $j$ and punctures $\left\{p_{k}\right\}$. Set $\dot{\Sigma}=\Sigma \backslash\left\{p_{k}\right\}$. For $r \in \mathbb{R}$ define the half-infinite cylinder

$$
C_{r}=[r, \infty) \times S^{1}
$$

with coordinates $s \in[r, \infty), t \in S^{1}=\mathbb{R} / \mathbb{Z}$ complex structure $j$ with $j \partial_{s}=\partial_{t}$ and volume form $d \mathrm{vol}=d s \wedge d t$. Set $C=C_{0}$. Then at each puncture $p_{k}$ we have local conformal coordinates $\sigma_{k}: C \rightarrow \dot{\Sigma}$.

Fix a constant $\delta>0$. Since we will be only interested in $\delta$ close to zero we assume throughout that $\delta$ is bounded by some constant $M$.

Definition 3.6 (Asymptotic energy) We say a map $v \in W_{\text {loc }}^{k, p}(C, Z)$ with $k p \geq 2$ has finite asymptotic energy with respect to a fixed closed characteristic and multiplicity $k \in \mathbb{Z}$ if there exists a parametrization $x \in \mathcal{R}$ of the closed characteristic so that

$$
E_{r}(v)=\int_{C_{r}}\left(d(v(s, t), x(k t))^{2}+|d v-k d x|^{2}\right) e^{\delta s} d \mathrm{vol}<\infty,
$$

where $d$ measures the distance in $Z$ with respect to $g_{Z}$.

This definition requires maps with finite asymptotic energy to converge to the closed characteristics exponentially fast at the punctures. 
In the end we are interested in $\mathcal{H}$-holomorphic maps (see Definition 4.1) from punctured Riemann surfaces. When restricted to half-infinite cylinders $C$ adjacent to a puncture the maps are actually $J$-holomorphic and solutions considered in the standard theory (eg [12] or [4]) have finite asymptotic energy as defined above.

Definition 3.7 Let $W_{\delta}^{k, p}(C, Z)$ be the space of finite asymptotic energy $W_{\text {loc }}^{k, p}(C, Z)$ maps with respect to a fixed closed characteristic. Similarly, let $W_{\delta}^{k, p}(\dot{\Sigma}, Z)$ be the space of $W_{\mathrm{loc}}^{k, p}(\dot{\Sigma}, Z)$ maps that are in $W_{\delta}^{k, p}(C, Z)$ in some local conformal coordinates at each puncture with respect to fixed closed characteristics.

Definition 3.8 For $v \in W_{\delta}^{k, p}(C, Z)$ let $W_{\delta}^{k, p}\left(C, v^{*} T Z\right)$ to be the space of sections $\zeta \in W_{\mathrm{loc}}^{k, p}\left(C, v^{*} T Z\right)$ that have finite asymptotic energy, ie such that there exists a constant $T^{\prime} \in \mathbb{R}$ so that

$$
\left.E_{r}(\zeta)=\left.\int_{C_{r}}\left(\mid \zeta-T^{\prime} R\right)\right|^{2}+|\nabla \zeta|^{2}\right) e^{\delta s} d \mathrm{vol}<\infty
$$

where $\nabla$ is the pullback of the Levi-Civita connection.

Analogously, for $v \in W_{\delta}^{k, p}(\dot{\Sigma}, Z)$ we define $W_{\delta}^{k, p}\left(\dot{\Sigma}, v^{*} T Z\right)$ to be the space of sections $\zeta \in W_{\mathrm{loc}}^{k, p}\left(\dot{\Sigma}, v^{*} T Z\right)$ that are in $W_{\delta}^{k, p}\left(C, v^{*} T Z\right)$ in local conformal coordinates at each puncture.

With these definitions $W_{\delta}^{k, p}(\dot{\Sigma}, Z)$ is a separable Banach manifold, modeled locally at a map $v \in W_{\delta}^{k, p}(\dot{\Sigma}, Z)$ on a neighborhood of the zero section in $W_{\delta}^{k, p}\left(\dot{\Sigma}, v^{*} T Z\right)$. For details we refer the reader to Dragnev [7] and Schwarz [22].

\subsection{Folded maps and folded holomorphic maps}

Definition 3.9 (Space of folded map) Fix a positive integer $k$ and $p \in \mathbb{R}$ with $k p>2$, nonnegative integers $g_{0}, g_{1}, n$ and relative homology classes $A_{ \pm} \in H_{2}\left(X_{ \pm}, \mathcal{R} ; \mathbb{Z}\right)$. Then a folded map $\left(u_{+}, u_{-}\right)$with respect to $A_{ \pm}$consists of

(i) a folded domain

(ii) $u_{ \pm} \in W^{k, p}\left(\Sigma_{ \pm}, X_{ \pm}\right)$

such that there exist maps $v_{ \pm} \in W_{\delta}^{k, p}(\dot{S}, Z)$ with

$$
u_{ \pm}^{*} \operatorname{det}(\omega)=\tau,\left.\quad u_{ \pm}\right|_{\sigma}=\left.v_{ \pm}\right|_{\sigma} \quad \text { and } \quad\left[u_{ \pm} \sqcup_{\sigma} v_{ \pm}\right]=A_{ \pm}
$$




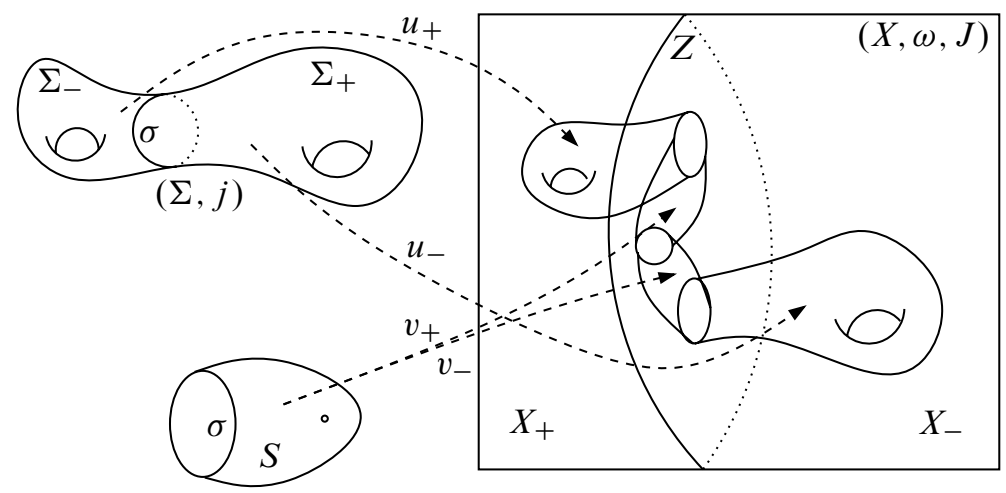

Figure 5: Folded (holomorphic) maps

Definition 3.10 (Folded holomorphic map) A folded map $\left(u_{+}, u_{-}\right)$is called folded holomorphic if $\bar{\partial}_{J} u_{ \pm}=0$ and $\left.\left(u_{+}, u_{-}\right)\right|_{\sigma} \in \Delta^{Z}$, where the folded diagonal $\Delta^{Z}$ is defined in Definition 4.7.

The key to defining folded holomorphic maps lies in the definition of the "matching condition". It is specified by the subspace

$$
\Delta^{Z} \subset \operatorname{Map}(\sigma, Z) \times \operatorname{Map}(\sigma, Z),
$$

called the folded diagonal. Intuitively, we view $\Delta^{Z}$ as a scattering function that takes boundary conditions of "incoming" holomorphic curves on the "+" side and transforms them into boundary conditions for "outgoing" holomorphic curves on the "-" side. To make sense out of this we don't need to define this scattering function for every element of $\operatorname{Map}(\sigma, Z)$ but it suffices to define it on the maps that are possible boundary conditions of holomorphic maps. The precise definition of the folded diagonal $\Delta^{Z}$ is rather involved and we postpone it until Section 4.1.

Lemma 3.11 The gauge group $\mathcal{G}$ defined in Definition 3.2 acts on the space of folded holomorphic maps by precomposition of $u_{ \pm}$by the element in $\operatorname{Diff}^{+}\left(\Sigma_{0}\right)$, on $v_{ \pm}$by the element in $\operatorname{Diff}^{+}\left(\Sigma_{1}\right)$ and acts on the folded domain as in Lemma 3.3.

Proof The action preserves solutions to the holomorphic map equation and the condition that $\tau_{0}=u_{ \pm}^{*} \operatorname{det}(\omega)$, since $\tau_{0}$ and $u_{ \pm}$are acted on by precomposition with the same diffeomorphism. Moreover it preserves the identification of the domain folds $\sigma_{0}$ and $\sigma_{1}$ as for $p \in \sigma$,

$$
u_{ \pm}^{\prime}(p)=u_{ \pm} \circ \phi_{0}(p)=v_{ \pm} \circ \psi \circ \phi_{0}(p)=v_{ \pm}^{\prime} \circ \phi_{1}^{-1} \circ \psi \circ \phi_{0}(p)=v_{ \pm}^{\prime} \circ \psi^{\prime}(p) .
$$


So this action preserves the set of folded holomorphic maps as long as it preserves the folded diagonal. We will postpone this part of the proof until Remark 4.8.

We make an important observation about the orientations of maps along the fold.

Remark 3.12 Let $p \in \sigma$ be a point where $\tau$ vanishes transversely and let $\eta \in T_{p} \Sigma$ be an outward normal vector to $\sigma$ at $p$, ie $j \eta \in T_{p} \sigma$ and $d \tau_{ \pm}(\eta)<0$. Recall the notation from Lemma 1.5. Remembering that $\tau_{ \pm}=u_{ \pm}^{*} \operatorname{det}(\omega)$ and $J_{ \pm} \partial_{r}= \pm R$ and thus $d r= \pm \alpha \circ J_{ \pm}$we have

$$
\begin{aligned}
u_{ \pm}^{*} \alpha(j \eta) & =\alpha\left(d u_{ \pm} j \eta\right)=\alpha \circ J_{ \pm}\left(d u_{ \pm} \eta\right)= \pm d r\left(d u_{ \pm}(\eta)\right)= \pm d(\operatorname{det}(\omega))\left(d u_{ \pm}(\eta)\right) \\
& = \pm d \tau_{ \pm}(\eta)
\end{aligned}
$$

so the values of $u_{ \pm}^{*} \alpha$ on tangent vectors have opposite sign.

This shows that at a point $p \in \sigma$ where $\tau_{ \pm}$vanish transversely, the gap-function $a$ from Equation (3.12) can be expressed in terms of $u_{ \pm}$using Equation (3.13) as

$$
a(p)=-\frac{u_{-}^{*} \alpha(p)}{u_{+}^{*} \alpha(p)}
$$

where the fraction is to be understood as evaluated on an arbitrary element of $T_{p} \Sigma$ such that the denominator does not vanish. This is well defined since $u_{ \pm}^{*} \alpha$ are collinear at $p$.

This turns out to be a crucial observation in showing that the folded diagonal poses an elliptic boundary condition and that therefore the linearized operator is Fredholm.

\section{Tunneling maps}

Throughout this section we assume that the function $\tau_{ \pm}$vanishes transversely, so the domain fold $\sigma$ is a manifold.

Tunneling maps give the matching conditions for pseudoholomorphic maps into $X_{ \pm}$, add homological data to the maps, and ensure that families of folded holomorphic maps have bounded energy. They are central in proving regularity of solutions and they guarantee that the linearized operator is Fredholm. In this section we define all relevant structures and discuss their properties. 


\subsection{Definition of tunneling maps and the folded diagonal}

Tunneling maps are maps into the fold $Z$ that connect the images of the folded maps into $X_{+}$and $X_{-}$. All tunneling maps will have domains $(\dot{S}, j)$ of the form $\left(\Sigma_{1}^{-} \backslash\{p\}, j_{1}\right)$ with boundary $\partial S=\sigma$.

Tunneling maps satisfy an equation that depends only on the CR structure $(Z, F, J)$ on the fold $Z$ and the 1 -form $\alpha$. First we need the following generalization of the holomorphic map equation.

Definition 4.1 ( $\mathcal{H}$-Holomorphic map) A map $v: \dot{S} \rightarrow Z$ is called $\mathcal{H}$-holomorphic if

$$
\begin{aligned}
\bar{\partial}_{J}^{F} v & =\frac{1}{2}\left(\pi_{F} d v+J \pi_{F} d v j\right)=0 \\
d\left(v^{*} \alpha \circ j\right) & =0 .
\end{aligned}
$$

$\mathcal{H}$-holomorphic maps can alternatively viewed as families of $J$-holomorphic maps into the complex cylinder ("symplectization") over $Z$ parametrized by

$$
\mathcal{H}_{n}(S, j)=\left\{\lambda \in \Omega^{1}(S) \mid d \lambda=0, d^{*} \lambda=0 \text { and } \lambda(j \eta)=0 \text { for all } \eta \in T \partial S\right\} .
$$

$\mathcal{H}_{n}$ is naturally isomorphic to $H^{1}(S, \mathbb{R})$ as a vector space and consists of smooth 1 -forms [8]. In [1], Abbas, Cieliebak and Hofer called maps from punctured surfaces without boundary satisfying this condition "generalized holomorphic".

Definition 4.2 (Tunneling map) Fix $k, p>0$ such that $k p>4$. A tunneling map is a $\mathcal{H}$-holomorphic map of class $W_{\delta}^{k, p}(\dot{S}, Z)$.

The choice $k p>4$ is made for convenience so that tunneling maps have a priori continuous derivatives on the boundary. On the interior they are smooth by elliptic regularity. In Theorem 6.2 we show that tunneling maps that give boundary values for folded holomorphic maps are also smooth on the boundary.

We make a simple, but essential observation about tunneling maps.

Lemma 4.3 Let $v$ be a tunneling map. Then the periods of $v^{*} \alpha \circ j$ vanish in a neighborhood of the puncture.

Proof Fix local conformal coordinates $C=[0, \infty) \times S^{1}$ at the puncture as in Definition 3.7. Since $v^{*} \alpha \circ j$ is closed, the value of

$$
\int_{\{r\} \times S^{1}} v^{*} \alpha \circ j=\int_{\{r\} \times S^{1}} v^{*} \alpha\left(\partial_{S}\right) d t
$$


does not depend on $r$. Observing that $A_{r}=(r, r+1) \times S^{1}$ has unit area and using Equation (3.15) we compute

$$
\begin{aligned}
\left|\int_{\{r\} \times S^{1}} v^{*} \alpha \circ j\right| & \leq \int_{A_{r}}\left|v^{*} \alpha\left(\partial_{s}\right)\right| d \mathrm{vol} \leq e^{-\delta r / 2}\left(\int_{A_{r}} e^{\delta s}\left|v^{*} \alpha\left(\partial_{s}\right)\right|^{2} d \mathrm{vol}\right)^{\frac{1}{2}} \\
& \leq e^{-\delta r / 2} \sqrt{E_{0}(v)}
\end{aligned}
$$

showing that the periods of $v^{*} \alpha \circ j$ are arbitrarily small and therefore vanish.

Tunneling maps are very well-behaved. They satisfy an elliptic system of PDEs and are therefore smooth on the interior of $\dot{S}$. Given elliptic boundary conditions their linearized operator is Fredholm for almost all choices of weight $\delta$ by arguments of [22] and [18, Theorem 6.3]. They have nice limits at the punctures, namely they converge to a closed characteristic exponentially fast as explained below. Thus they extend to continuous maps from the oriented blowup of the domain. We will blur the distinction between a tunneling map and its continuous extension to the oriented blowup.

To understand how tunneling maps approach a parametrized closed characteristic let $v: C=[0, \infty) \times S^{1} \rightarrow Z$ be the restriction of a tunneling map to a conformal neighborhood of a puncture and assume that $v$ approaches a parametrized closed characteristic $x$ with minimal period $T>0$ and multiplicity $k \in \mathbb{Z}$, ie

$$
\lim _{s \rightarrow \infty} v(s, t)=x_{k}(t)=x(k t)=\phi_{k T t}(x(0))
$$

where $\phi$ is the characteristic flow. In light of Lemma 4.3, $v^{*} \alpha \circ j$ is exact and thus this restriction is $J$-holomorphic. Therefore the asymptotic convergence results of [12] and [4] carry over verbatim for tunneling maps. In particular they apply to the transverse approach to a closed characteristic by studying the map in a tubular neighborhood of the closed characteristic $x$. Let

$$
\phi: x^{*} F \rightarrow Z, \quad \phi(\eta)=\exp _{x(\theta)}(\eta(\theta))
$$

be a local diffeomorphism of a neighborhood of the zero section of $x^{*} F$ onto a tubular neighborhood of the image of $x$ in $Z$. Using this we define the family of sections

$$
\eta(s)=\phi^{-1}(v(s, \cdot))
$$

for $s$ large enough. Since $v(s, t)$ approaches $x_{k}(t)$ exponentially fast,

$$
\|\eta(s)\|^{2}=\int_{S^{1}} \omega(J \eta(s)(t), \eta(s)(t)) d t
$$


approaches 0 exponentially fast. To understand this approach we view $\eta$ as a family of sections of $x_{k}^{*} F$ for $s$ big enough, and consider the renormalized family of sections

$$
\xi(s)=\eta(s) /\|\eta(s)\| .
$$

Since $\eta$ satisfies a differential equation, so does $\xi$, and in the limit as $s \rightarrow \infty, \xi(s)$ approaches a section $\xi_{0}$ of the $k$-fold cover $x_{k}^{*} F$ of $x^{*} F$ that is an eigenvector of the asymptotic operator

$$
A_{\infty}: W^{1,2}\left(x_{k}^{*} F\right) \subset L^{2}\left(x_{k}^{*} F\right) \rightarrow L^{2}\left(x_{k}^{*} F\right), \quad A_{\infty}(\xi)=-k T J \mathcal{L}_{R} \xi
$$

corresponding to an eigenvalue smaller or equal to $-\delta / 2$, where $\delta$ is the weight from the asymptotic energy (3.15). For details we refer the reader to [12], [4] and [1].

Remark 4.4 Note that the asymptotic energy (3.15) is invariant under diffeomorphisms of the domain, so the gauge group $\mathcal{G}$ from Definition 3.2 acts on the space of tunneling maps by precomposition.

Tunneling maps connect boundary values of $J$-holomorphic maps in $X_{ \pm}$to closed characteristics. Given a map $\Gamma: \sigma \rightarrow Z$ that is a boundary value of a $J$-holomorphic map into $X_{+}$or $X_{-}$, we can ask whether there exists a tunneling map $v: \dot{S} \rightarrow Z$ with $\left.v\right|_{\sigma}=\Gamma$. In the circle-invariant case existence of such a map is equivalent to existence of a $J$-holomorphic map $\widetilde{v}: S \rightarrow V$ to the base of the $S^{1}$-bundle $V$ with boundary on $\pi_{V} \Gamma$. Since domain and target are 2-dimensional, this question is well understood if the image of $\Gamma$ under the projection $\pi_{V}$ is sufficiently nice and only depends on topological data. Any such map lifts to a unique $\mathcal{H}$-holomorphic map in each relative homotopy class of lifts by the same arguments as in Remark 4.6.

\subsection{Conjugate tunneling maps}

To get a scattering function of incoming boundary values from a map $u_{+}$into $X_{+}$to outgoing boundary values of a map $u_{-}$into $X_{-}$, we need to define a relation between tunneling maps $v_{+}$connecting to $u_{+}$and $v_{-}$connecting to $u_{-}$.

We clarify some notation used in this section. For a tunneling map $v$ and an asymptotic marker $m \in\left(T_{p} S \backslash 0\right) / \mathbb{R}^{>0}$ in boundary $\widehat{S} \backslash \dot{S}$ of the oriented blowup $\widehat{S}$ of $\dot{S}$ we have that $v(m)$ has image on some closed characteristic $x \in \mathcal{R}$. Given a parametrization $x(t): S^{1} \rightarrow Z$ of the closed characteristic, it inherits the group structure from $S^{1}=\mathbb{R} / \mathbb{Z}$ via $x(s)+x(t)=x(s+t)$.

Definition 4.5 (Conjugate tunneling map) Two tunneling maps $v_{+}$and $v_{-}$with domain $(\dot{S}, j)$ that are asymptotic to the same closed characteristic $x \in \mathcal{R}$ are called 
conjugate with respect to a parametrization $x(t)$ of the closed characteristic if there exists a function $\rho: S \rightarrow(0, \infty)$ with $\left.\rho\right|_{\sigma} \equiv 1$ such that

$$
\begin{aligned}
v_{+}^{*} \omega & =\rho v_{-}^{*} \omega & & \text { on } \dot{S} \\
\lambda=v_{+}^{*} \alpha \circ j+v_{-}^{*} \alpha \circ j & =0 & & \text { on } T \sigma \\
v_{+}(m)+v_{-}(m) & =x(0) & & \text { for all } m \in \hat{S} \backslash \dot{S} .
\end{aligned}
$$

Equation (4.22) implies that $v_{ \pm}$have transverse approach to eigenvectors corresponding to the same eigenvalue of the asymptotic operator (4.21). If the eigenvalue is not simple we also demand that $v_{+}$and $v_{-}$have transverse approach to the same eigenvector.

Let

$$
Q=\left\{q \in S \mid v_{ \pm} \text {fails to be an immersion at } q\right\} .
$$

In the case of $S^{1}$-invariant folds we further demand that the order of vanishing of the 1 -form $\lambda$ defined in (4.23) is no less than the one of $\pi_{F} d v_{ \pm}$at each point $q \in Q$.

Remark 4.6 If $v_{+}$and $v_{-}$are conjugate, then their projections $\tilde{v}_{ \pm}=\pi_{V} \circ v_{ \pm}$to the base $V$ agree by Equation (4.22) and the requirement that $v_{ \pm}$have transverse approach to the same eigenvector of the asymptotic operator. To see this look at the projections $\widetilde{v}_{ \pm}=\pi_{V} v_{ \pm}$. By Equation (4.22) their differentials satisfy $d \widetilde{v}_{+}=\tilde{\rho} \cdot \tilde{d} v_{-}$ on $S$ for some nowhere vanishing holomorphic function $\tilde{\rho}: S \rightarrow \mathbb{C}$. Since the function $\rho$ in Equation (4.22) equals 1 on $\sigma$ we conclude that $\left.\tilde{\rho}\right|_{\sigma}: \sigma \rightarrow S^{1}$ takes values in the unit circle. Since $\tilde{\rho}$ has degree 0 we conclude that $\tilde{\rho}$ is constant. $v_{ \pm}$approach the same closed characteristic, so $\widetilde{v}_{+}(p)=\widetilde{v}_{-}(p)$. The requirement that the transverse components converge to the same eigenvector of the asymptotic operator simply means that $d \widetilde{v}_{+}(p)=d \widetilde{v}_{-}(p)$, so $\tilde{\rho}(p)=1$. Thus $\tilde{\rho}=1$ and $\widetilde{v}_{+}=\tilde{v}_{-}$.

Thus there exists an $S^{1}$-valued function

$$
g: \dot{S} \rightarrow S^{1} \quad \text { with } v_{-}(z)=g(z) * v_{+}(z),
$$

where $*$ denotes the $S^{1}$-action on $Z$ given by the characteristic flow. Equation (4.24) implies that $v_{+}$and $v_{-}$have opposite multiplicities at the punctures and the degree of $g$ at the puncture is $-2 d$ if $d$ is the degree of $v_{+}$at the puncture.

The function $g$ satisfies $d g=v_{-}^{*} \alpha-v_{+}^{*} \alpha$ so

$$
\begin{aligned}
& d^{*} d g=* d(d g \circ j)=* d\left(v_{-}^{*} \alpha \circ j-v_{+}^{*} \alpha \circ j\right)=0, \\
& d g \circ j=-2 v^{*} \alpha \circ j \quad \text { on } T \partial S,
\end{aligned}
$$

where we used Equations (4.20) and (4.23). Therefore $g$ is a harmonic $S^{1}$-valued function with prescribed asymptotics satisfying von Neumann boundary conditions. 
This determines $g$ uniquely up to $H^{1}(S ; \mathbb{Z})$ and an overall constant. Equation (4.24) fixes the overall constant, leaving us with a discrete set of choices. By Remark 3.12 we conclude that only a finite number, bounded by a constant only depending on $d$, of values for the periods of $d g$ can appear as boundary values for $J$-holomorphic maps into $X_{ \pm}$, at least for generic domains $(S, j)$. To see this fix a set of generators of $\mathcal{H}_{n}(S ; \mathbb{Z})$ and consider their effect on $v_{-}$and consequently the gap-function from Equation (3.18).

In the circle-invariant case the zeros of $\pi_{F} d v_{ \pm}$automatically agree. Thus the dimension of the space of conjugate tunneling maps jumps as the tunneling maps acquire points $q \in Q$. To deal with this nongeneric situation we introduced the additional restriction on the order of vanishing of $\lambda$ along $Q$.

We adopt the convention that for folded maps $\left(u_{+}, u_{-}\right)$and tunneling maps $\left(v_{+}, v_{-}\right)$ their restrictions to $\sigma$ are denoted by a hat, ie

$$
\widehat{u}_{ \pm}=\left.u_{ \pm}\right|_{\sigma} \quad \text { and } \quad \hat{v}_{ \pm}=\left.v_{ \pm}\right|_{\sigma} .
$$

We are now prepared for the main definition.

Definition 4.7 (Folded diagonal) The folded diagonal is the subset in the product $\operatorname{Map}(\sigma, Z) \times \operatorname{Map}(\sigma, Z)$ defined by

$$
\Delta^{Z}=\left\{\left(\hat{v}_{+}, \widehat{v}_{-}\right) \mid\left(v_{+}, v_{-}\right) \text {are conjugate tunneling maps }\right\} .
$$

Remark 4.8 Note that the folded diagonal is invariant under the action of the gauge group $\mathcal{G}$. Indeed, if $\left(v_{+}, v_{-}, j\right)$ is a conjugate pair of tunneling maps, and $\phi_{1}: \Sigma_{1} \rightarrow \Sigma_{1}$ is a diffeomorphism, then $\left(\phi_{1}^{*} v_{+}, \phi_{1}^{*} v_{-}, \phi_{1}^{*} j\right)$ is also a pair of conjugate tunneling maps with domain $\left(\phi_{1}^{*} \dot{S}, \phi_{1}^{*} j_{1}\right)$. This concludes the proof of Lemma 3.11.

\section{Properties of the folded diagonal}

In this section we study the properties of the folded diagonal by looking at its deformation space. In short, we find that the deformations of the folded diagonal at a pair of conjugate tunneling maps restricted to $\sigma\left(\hat{v}_{+}, \hat{v}_{-}\right)$is given by the graph of a function from sections of $\hat{v}_{+}^{*} T Z$ to sections of $\hat{v}_{-}^{*} T Z$, which we will describe.

We only consider the case of $S^{1}$-invariant folds. They have the advantage that the $\mathcal{H}$-holomorphic map equations are linear and upper triangular, which simplifies the proofs. Moreover, in the $S^{1}$-invariant case most of the proofs are constructive which provides further intuitive insight into the properties of folded holomorphic maps. 
First we give the linearization of the equation for tunneling maps. As in the definition of the space of maps, we use a metric contact connection. There are many choices for such connections; see Nicolaescu [21]. In the circle-invariant case a choice that is particularly convenient is the Levi-Civita connection $\nabla$ of the metric $g_{Z}$. One easily checks that $\nabla$ is contact metric, ie $\nabla \alpha=\nabla \omega=0$. The reason for this is that the associated canonical complex structure on the symplectization is integrable. Consequently we also have $\nabla R=\nabla J=\nabla \pi_{F}=0$.

Lemma 5.1 At a solution (v,j), the linearizations of equations (4.19) and (4.20) are

where

$$
\begin{aligned}
D_{(v, j)}^{F}(\xi, h) & =\pi_{F} \nabla^{0,1} \xi+\frac{1}{2} J \pi_{F} d v h=0 \\
D_{(v, j)}^{L}(\xi, h) & =d\left[d(\alpha(\xi)) \circ j+v^{*}(\iota \xi d \alpha) \circ j+v^{*} \alpha \circ h\right]=0 \\
\nabla^{0,1} & =\frac{1}{2}\{\nabla+J \nabla \circ j\} .
\end{aligned}
$$

Proof Let $v_{t}$ be a family of tunneling maps with complex structures $j_{t}, \xi=\left.\frac{d}{d t}\right|_{t=0} v_{t}$ and $h=\left.\frac{d}{d t}\right|_{t=0} j$. Then

$$
\begin{aligned}
D_{(v, j)}^{F}(\xi, h) & =\left.\frac{d}{d t}\right|_{t=0} \frac{1}{2} \pi_{F}\left\{d v_{t}+J d v_{t} j_{t}\right\} \\
& =\frac{1}{2} \pi_{F}\left\{\nabla_{t} d v_{t}+J \nabla_{t}\left(d v_{t}\right) \circ j+J d v h\right\}_{t=0} \\
& =\pi_{F} \nabla^{0,1} \xi+\frac{1}{2} J \pi_{F} d v h .
\end{aligned}
$$

For the second equation we compute

$$
\left.\frac{d}{d t}\right|_{t=0} v_{t}^{*} \alpha \circ j_{t}=\left(v^{*} \mathcal{L}_{\xi} \alpha\right) \circ j+v^{*} \alpha \circ h=d(\alpha(\xi)) \circ j+v^{*}\left(\iota_{\xi} d \alpha\right) \circ j+v^{*} \alpha \circ h .
$$

So the system of PDEs (5.26) and (5.27) is upper triangular with respect to the splitting $T Z=L \oplus F$. This greatly simplifies computations and the construction of examples.

Definition 4.5 allows us to define useful bundle homomorphisms.

Definition 5.2 Let $v_{+}$and $v_{-}$be conjugate tunneling maps. Then define the $\left(J_{+}, J_{-}\right)-$ linear vector bundle isomorphisms

$$
\begin{array}{ll}
A^{F}: v_{+}^{*} F \rightarrow v_{-}^{*} F, & A^{F}=\left(\pi_{F} d v_{-}\right) \circ\left(\pi_{F} d v_{+}\right)^{-1} \\
A^{E}: v_{+}^{*} E \rightarrow v_{-}^{*} E & A^{E}\left(\zeta_{1} \partial_{r}+\zeta_{2} R\right)=\zeta_{1} \partial_{r}-\zeta_{2} R .
\end{array}
$$

The isomorphism $A^{F}$ is well defined at the zeros of $\pi_{F} d v_{+}$by Equation (4.22). 
By the vanishing condition on $\lambda$ along $Q$ that we imposed in the case of circle-invariant folds at the end of Definition 4.5 we may define the linear map

$$
f: v_{+}^{*} F \rightarrow \mathbb{R}, \quad f=\lambda \circ\left(\pi_{F} d v_{+}^{-1}\right) .
$$

Remark 5.3 In the following we will assume that, unless $\alpha$ is closed, at each point $q \in Q$ the map

$$
(\nabla f)(q): F_{v_{ \pm}(q)} \rightarrow T_{q}^{*} S
$$

is surjective and that the order of vanishing of $\pi_{F} d v_{ \pm}$is no greater than 1 .

This assumption holds generically. The projection of $v_{ \pm}$to the base $V$ of the $S^{1}-$ bundle $Z$ is holomorphic, so given elliptic boundary conditions, the stratum with zeros of order $\geq 2$ has codimension at least 2 . The condition that, given $\pi_{F} v_{ \pm}, \nabla f$ not vanish holds for generic boundary conditions.

Lemma 5.4 Let $\left(\xi_{+}, h\right)$ and $\left(\xi_{-}, h\right)$ be deformations of the conjugate tunneling maps $v_{+}$and $v_{-}$The linearizations of the equations for conjugate tunneling maps (4.22) through (4.24) are

$$
\begin{gathered}
\pi_{F} \xi_{+}=\pi_{F} \xi_{-} \\
d\left[\alpha\left(\xi_{+}\right)+\alpha\left(\xi_{-}\right)\right] \circ j=\lambda \circ j h-\left[v_{+}^{*}\left(\iota_{+} d \alpha\right)+v_{-}^{*}\left(\iota_{-} d \alpha\right)\right] \circ j \quad \text { on } T \sigma \\
\alpha\left(\xi_{+}\right)(p)+\alpha\left(\xi_{-}\right)(p)=0 .
\end{gathered}
$$

Proof This follows immediately from the definitions and Remark 4.6.

We need the following technical lemmas.

Lemma 5.5 Let $\left(\Sigma, \sigma, j, u_{+}, u_{-}\right)$be a folded holomorphic map with conjugate tunneling maps $\left(\dot{S}, j, v_{+}, v_{-}\right)$. Then for $\eta \in T_{\sigma} S=T_{\sigma} \Sigma$,

$$
\lambda(\eta)=v_{+}^{*} \alpha(j \eta)+v_{-}^{*} \alpha(j \eta)=u_{+}^{*} \alpha(j \eta)+u_{-}^{*} \alpha(j \eta) .
$$

Proof The first equality is just the definition of $\lambda$ in Equation (4.23). Recall that $\widehat{u}_{ \pm}=\widehat{v}_{ \pm}$and both $d u_{ \pm}$and $\pi_{F} d v_{ \pm}$are $\left(j, J_{ \pm}\right)$-linear. Therefore

$$
\pi_{F} d u_{ \pm}=\pi_{F} d v_{ \pm} \quad \text { over } \sigma
$$

For $\eta \in T \sigma$ we have

$$
u_{ \pm}^{*} \alpha(\eta)=v_{ \pm}^{*} \alpha(\eta), \quad u_{ \pm}^{*} \alpha(j \eta)=0, \quad v_{+}^{*} \alpha(j \eta)=-v_{-}^{*} \alpha(j \eta),
$$

where the second equation holds since $\pi_{E} d u_{ \pm}$is $J_{ \pm}$-linear and $\left.\pi_{K} d u_{ \pm}\right|_{T \sigma}=0$ and the last equation is just Equation (4.23). Equation (5.33) follows. 
Lemma 5.6 Let $v$ be a tunneling map, $\eta$ a section of $T S$ vanishing at the puncture, and $h$ a deformation of complex structure $j$ such that

$$
\nabla^{0,1} \eta+\frac{1}{2} j h=0
$$

Then

$$
d\left(v^{*} \alpha(j \eta)\right)-d\left(v^{*} \alpha(\eta)\right) \circ j=\left(\iota_{\eta} v^{*} d \alpha\right) \circ j+v^{*} \alpha \circ h
$$

and consequently, if $v_{+}$and $v_{-}$are conjugate tunneling maps,

$$
d[\lambda(\eta)]+d[\lambda(j \eta)] \circ j=\left(\iota_{\eta}\left[v_{+}^{*} d \alpha+v_{-}^{*} d \alpha\right]\right) \circ j-\lambda \circ j h .
$$

Proof Near some point $z \in \dot{S}$, let $X$ be a section of $T S$ with $\nabla X(z)=0$.

$$
\begin{aligned}
d\left(v^{*} \alpha(\eta)\right) \circ j(X) & =\mathcal{L}_{j X}\left(v^{*} \alpha(\eta)\right) \\
& =\mathcal{L}_{j X}\left(v^{*} \alpha\right)(\eta)+v^{*} \alpha\left(\nabla_{j X} \eta\right) \\
& =\mathcal{L}_{\eta}\left(v^{*} \alpha(j X)\right)+v^{*} d \alpha(j X, \eta)+v^{*} \alpha\left(j \nabla_{X} \eta-h(X)\right) \\
& =\mathcal{L}_{\eta}\left(v^{*} \alpha \circ j\right)(X)-v^{*} d \alpha(\eta, j X)-v^{*} \alpha \circ h(X) \\
& =\left[d\left(v^{*} \alpha(j \eta)\right)-\left(\iota_{\eta} v^{*} d \alpha\right) \circ j-v^{*} \alpha \circ h\right](X) .
\end{aligned}
$$

Lemma 5.7 Let $\left(v_{+}, v_{-}, j\right)$ be a pair of conjugate tunneling maps and, $\xi$ a section of $v_{+}^{*} T Z$ and $h$ a deformation of complex structure $j$ such that

$$
D_{\left(v_{+}, j\right)}^{F}(\xi, h)=0
$$

Then, with $\chi=\pi_{F} \xi$,

$$
d[f(\chi)]+d[f(J \chi)] \circ j=\left[v_{+}^{*}\left(\iota_{\chi} d \alpha\right)+v_{-}^{*}\left(\iota_{A^{F}(\chi)} d \alpha\right)\right] \circ j-\lambda \circ j h .
$$

Proof Note that $f$ is a smooth function, so it suffices to show this on the dense subset $\ddot{S}=\dot{S} \backslash Q \subset \dot{S}$. On $\ddot{S}$ we may write $\chi=\pi_{F} d v_{+}(\eta)$.

Note that on $\ddot{S}$,

$$
D_{\left(v_{+}, j\right)}^{F}(\xi, h)=0 \quad \text { if and only if } \quad \nabla^{0,1} \eta+\frac{1}{2} j h=0 .
$$

Using Lemma 5.6 and the fact that $\iota_{R} d \alpha=0$,

$$
\begin{aligned}
d[f(\chi)]+d[f(J \chi)] \circ j & =d[\lambda(\eta)]+d[\lambda(j \eta)] \circ j \\
& =\left(\iota_{\eta}\left[v_{+}^{*} d \alpha+v_{-}^{*} d \alpha\right]\right) \circ j-\lambda \circ j h \\
& =\left[v_{+}^{*}\left(\iota_{\chi} d \alpha\right)+v_{-}^{*}\left(\iota_{A^{F}(\chi)} d \alpha\right)\right] \circ j-\lambda \circ j h,
\end{aligned}
$$

so Equation (5.36) holds on $\ddot{S}$ and by smoothness of $f$ it holds on $\dot{S}$. 


\subsection{Deformations of the folded diagonal}

Using the results of the previous section we describe the deformations of the folded diagonal. We show that they are given by the graph of a pseudodifferential operator $B^{Z}: \Gamma\left(\hat{u}_{+}^{*} T Z\right) \rightarrow \Gamma\left(\hat{u}_{-}^{*} T Z\right)$.

Theorem 5.8 Given conjugate tunneling maps $\left(v_{+}, v_{-}, j\right)$, a section $\hat{\xi}$ of $\hat{v}_{+}^{*} T Z$. Then there exists an extension $\xi_{+}$of $\widehat{\xi}$ to $v_{+}^{*} T Z$ a section $\xi_{-}$of $v_{-}^{*} T Z$ and a deformation $h$ of the complex structure $j$ so that $\left(\xi_{ \pm}, h\right)$ satisfy Equations (5.26) and (5.27) and $\left(\xi_{+}, \xi_{-}, h\right)$ satisfies Equations (5.30) through (5.32) and preserving the vanishing condition on $\lambda$ along the set of nonimmersion points $Q$ as given at the end of Definition 4.5.

Moreover the restriction $\left.\xi_{-}\right|_{\sigma}$ is uniquely determined by $\hat{\xi}$.

Proof We split this argument into the two cases when $\alpha$ is closed and when $\alpha$ is not closed.

If $\alpha$ is not closed, recall the Equation (5.28) defining the function $f: v_{ \pm}^{*} F \rightarrow \mathbb{R}$. Let $g: S \rightarrow \mathbb{R}$ be the unique harmonic function vanishing at the puncture and satisfying von Neumann boundary conditions

$$
d g \circ j=d\left[f\left(\pi_{F} \hat{\xi}\right)\right] \quad \text { on } T \sigma .
$$

Let $\xi$ be any extension of $\hat{\xi}$ to $\dot{S}$ that vanishes in a neighborhood of the puncture, satisfies

$$
(\nabla f)\left(\pi_{F} \xi\right)=d g \circ j \quad \text { on } Q
$$

and $\pi_{F} \nabla^{0,1} \xi=0$ in a neighborhood of $Q$. This is well-defined by the assumption that $\left.f\right|_{Q}: F_{v(Q)} \rightarrow T_{Q}^{*} S$ is surjective (see Remark 5.3).

Then we may set

$$
h=2\left(\pi_{F} d v_{+}\right)^{-1}\left(J \pi_{F} \nabla^{0,1} \xi\right)
$$

which is $j$-antilinear, and consequently $D_{\left(v_{+}, j\right)}^{F}(\xi, h)=0$. Now let $\zeta: S \rightarrow \mathbb{R}$ be the unique solution to the Dirichlet problem

$$
\begin{aligned}
d(d \zeta \circ j) & =-d\left[d(\alpha(\xi)) \circ j+v_{+}^{*}(\iota \xi d \alpha) \circ j+v_{+}^{*} \alpha \circ h\right], \\
\left.\zeta\right|_{\sigma} & =0 .
\end{aligned}
$$


Then $\xi_{+}=\xi+\zeta \cdot R$ satisfies

$$
\begin{aligned}
D_{\left(v_{+}, j\right)}^{F}\left(\xi_{+}, h\right) & =D_{\left(v_{+}, j\right)}^{F}(\xi, h)=0, \\
D_{\left(v_{+}, h\right)}^{L}\left(\xi_{+}, h\right) & =D_{\left(v_{+}, j\right)}^{L}(\xi, h)+d(d \zeta \circ j)=0, \\
\left.\xi_{+}\right|_{\sigma} & =\hat{\xi} .
\end{aligned}
$$

Now define the section $\xi_{-}$of $v_{-}^{*} T Z$ by

$$
\xi_{-}=A^{F}\left(\pi_{F} \xi_{+}\right)-\left(f\left(J \pi_{F} \xi_{+}\right)+\alpha\left(\xi_{+}\right)+g\right) R .
$$

Then $D_{\left(v_{-}, j\right)}^{F}\left(\xi_{-}, h\right)=D_{\left(v_{+}, j\right)}^{F}\left(\xi_{+}, h\right)=0$

$$
\text { and } \begin{aligned}
D_{\left(v_{-}, j\right)}^{L}\left(\xi_{-}, h\right)= & d\left[d\left[\alpha\left(\xi_{-}\right)\right] \circ j+v_{-}^{*}\left(\iota_{-} d \alpha\right) \circ j+v_{-}^{*} \alpha \circ h\right] \\
= & d\left[-d\left[f\left(J \pi_{F} \xi_{+}\right)+\alpha\left(\xi_{+}\right)+g\right] \circ j+v_{-}^{*}\left(\iota_{-} d \alpha\right) \circ j\right. \\
& \left.\quad-\lambda \circ j h-v_{+}^{*} \alpha \circ h\right] \\
= & d\left[-d\left[f\left(J \pi_{F} \xi_{+}\right)\right] \circ j+v_{+}^{*}\left(\iota \xi_{+} d \alpha\right) \circ j+v_{-}^{*}\left(\iota \xi_{-} d \alpha\right) \circ j\right. \\
& \quad-\lambda \circ j h] \\
= & d\left[d\left(f\left(\pi_{F} \xi_{+}\right)\right)\right] \\
= & 0
\end{aligned}
$$

where we used Equation (5.36) in the second-to-last step.

Now we need to show that $\left(\xi_{+}, \xi_{-}, h\right)$ is a deformation of conjugate tunneling maps. By construction $\pi_{F} \xi_{-}=A^{F}\left(\pi_{F} \xi_{+}\right)$, verifying Equation (5.30). For Equation (5.31) we compute on $T \sigma$,

$$
\begin{aligned}
d\left[\alpha\left(\xi_{+}\right)+\alpha\left(\xi_{-}\right)\right] \circ j= & -d\left[f\left(J \pi_{F} \xi_{+}\right)+g\right] \circ j \\
= & -d\left[f\left(J \pi_{F} \xi_{+}\right)\right] \circ j-d g \circ j \\
= & d\left[f\left(\pi_{F} \xi_{+}\right)\right]-d g \circ j-\left[v_{+}^{*}\left(\iota_{\xi_{+}} d \alpha\right)+v_{-}^{*}\left(\iota_{-} d \alpha\right)\right] \circ j \\
& +\lambda \circ j h \\
= & d\left[f\left(\pi_{F} \hat{\xi}\right)\right]-d g \circ j-\left[v_{+}^{*}\left(\iota_{\xi_{+}} d \alpha\right)+v_{-}^{*}\left(\iota_{\xi_{-}} d \alpha\right)\right] \circ j \\
& +\lambda \circ j h \\
= & \lambda \circ j h-\left[v_{+}^{*}\left(\iota_{\xi_{+}} d \alpha\right)+v_{-}^{*}\left(\iota_{\xi_{-}} d \alpha\right)\right] \circ j
\end{aligned}
$$

where we used Equations (5.36) and (5.37). For Equation (5.32) we compute

$$
\alpha\left(\xi_{+}\right)(p)+\alpha\left(\xi_{-}\right)(p)=f\left(J_{\pi_{F}} \xi_{+}(p)\right)+g(p)=f\left(J \pi_{F} \xi(p)\right)=0 .
$$


We are left to verify that the condition on the order of vanishing of $\lambda$ along $Q$ is preserved. Linearizing $\left.\pi_{F} d v_{ \pm}\right|_{Q}=0$ gives

$$
\pi_{F} \nabla_{v} \pi_{F} d v_{ \pm}+\pi_{F} \nabla\left(\pi_{F} \xi_{ \pm}\right)=0 \quad \text { at all } q \in Q
$$

where $v$ is the deformation of $q$. Note that $\pi_{F} \nabla_{\nu}\left(\pi_{F} d v_{ \pm}\right)=\pi_{F} \nabla\left(\pi_{F} d v_{ \pm}(v)\right)$ at $q$, so we conclude that

$$
\pi_{F} \nabla\left(\pi_{F}\left(d v_{ \pm}(v)+\xi_{ \pm}\right)\right)=0 \quad \text { at all } q \in Q .
$$

Linearizing the condition that $\lambda$ vanishes on $Q$ gives

$$
\nabla_{\nu} \lambda+\left[v_{+}^{*}\left(\iota_{+} d \alpha\right)+v_{-}^{*}\left(\iota_{\xi_{-}} d \alpha\right)\right] \circ j+d\left[\alpha\left(\xi_{+}\right)+\alpha\left(\xi_{-}\right)\right] \circ j-\lambda \circ j h=0 .
$$

Using Equation (5.36) we see that

$$
\begin{aligned}
{\left[v_{+}^{*}\left(\iota_{+} d \alpha\right)\right.} & \left.+v_{-}^{*}\left(\iota_{\xi_{-}} d \alpha\right)\right] \circ j+d\left[\alpha\left(\xi_{+}\right)+\alpha\left(\xi_{-}\right)\right] \circ j-\lambda \circ j h \\
& =\left[v_{+}^{*}\left(\iota_{\xi_{+}} d \alpha\right)+v_{-}^{*}\left(\iota_{\xi_{-}} d \alpha\right)\right] \circ j-d\left[f\left(J \pi_{F} \xi_{ \pm}\right)\right] \circ j-d g \circ j-\lambda \circ j h \\
& =d\left[f\left(\pi_{F} \xi_{ \pm}\right)\right]-d g \circ j .
\end{aligned}
$$

Using that $\nabla_{v} \lambda=d(\lambda(v))=d\left[f\left(d v_{ \pm}(v)\right)\right]$ on $Q$ we see that on $Q$,

$$
\begin{aligned}
\nabla_{\nu} \lambda+d\left[f\left(\pi_{F} \xi_{ \pm}\right)\right]-d g \circ j & =\nabla\left(f\left[\pi_{F}\left(\xi_{ \pm}+d v_{ \pm}(v)\right)\right]\right)-d g \circ j \\
& =(\nabla f)\left(\pi_{F}\left[\xi_{ \pm}+d v_{ \pm}(v)\right]\right)-d g \circ j \\
& =(\nabla f)\left(\pi_{F} \xi_{ \pm}\right)-d g \circ j \\
& =0
\end{aligned}
$$

where we used Equation (5.40) in the third last step and Equation (5.38) in the last step. Combining this with the previous computation verifies Equation (5.41).

This establishes existence. To see uniqueness, suppose that $\left(\xi_{+}^{\prime}, h^{\prime}\right)$ also satisfies $D_{\left(v_{+}, j\right)}\left(\xi_{+}^{\prime}, h^{\prime}\right)=0$ and $\left.\xi_{+}^{\prime}\right|_{\sigma}=\widehat{\xi}$. Then $\left(\xi^{\prime \prime}=\xi_{+}-\xi_{+}^{\prime}, h^{\prime \prime}=h-h^{\prime}\right)$ satisfies

$$
D\left(\xi^{\prime \prime}, h^{\prime \prime}\right)=0,\left.\quad \xi^{\prime \prime}\right|_{\sigma}=0 .
$$

The above argument shows that given $\left(\xi_{+}, h\right)$ there is a unique pair $\left(\xi_{-}, h\right)$ satisfying Equations (5.26) and (5.27) so that $\left(\xi_{+}, \xi_{-}, h, \mu\right)$ satisfy Equations (5.30) through (5.32). In order that the condition on the vanishing of $\lambda$ is preserved we need that, with the above notation, $f\left(J \pi_{F} \xi_{+}\right)=d g \circ j$ on $Q$. Thus we conclude that

$$
\pi_{F} \xi^{\prime \prime}=0 \quad \text { on } Q
$$

so there exists a vector field $\eta$ on $S$ vanishing on $\sigma$ so that $\pi_{F} \xi^{\prime \prime}=\pi_{F} d v_{+}(\eta)$. Since $\pi_{F} d v_{+}$is $(j, J)$ linear and $\nabla J=0$ we conclude that $\nabla^{0,1} \eta+\frac{1}{2} j h^{\prime \prime}=0$. The 
function $\zeta^{\prime \prime}=\alpha\left(\xi^{\prime \prime}-d v_{+}(\eta)\right)$ vanishes on $\sigma$ and satisfies

$$
d\left(d \zeta^{\prime \prime} \circ j\right)=0
$$

so $\zeta^{\prime \prime}=0$ and $\xi^{\prime \prime}=d v_{+}(\eta)$. Thus $\left(\xi_{+}, h\right)$ and $\left(\xi_{+}^{\prime}, h^{\prime}\right)$ differ by an infinitesimal gauge transformation $\eta$. By Remark 4.8 this gauge ambiguity preserves the conjugacy condition and does not change the boundary values $\left.\xi_{ \pm}\right|_{\sigma}$.

Now consider the case when $d \alpha=0$. In this case $Z$ is a trivial $S^{1}$-bundle over $V$ and the two Equations (5.26) and (5.27) decouple and we may solve each one separately. This implies that $v_{+}^{*} \alpha+v_{-}^{*} \alpha \equiv 0$, so $\lambda \equiv 0$. As before, for given boundary values $\hat{\xi} \in \Gamma\left(\hat{v}_{+}^{*} T Z\right)$ there exist extensions $\xi_{+}$to sections of $v_{+}^{*} T Z$ and deformation $h$ of complex structure $j$ so that Equations (5.26) and (5.27) are satisfied. Then

$$
\xi_{-}=A^{F}\left(\pi_{F} \xi_{+}\right)-\alpha\left(\xi_{+}\right) R
$$

is the unique section of $v_{-}^{*} T Z$ satisfying Equations (5.26), (5.27) and (5.30) through (5.32). But $\left.\xi_{-}\right|_{\sigma}$ only depends on $\left.\xi_{+}\right|_{\sigma}$, so all possible choices of $\xi_{+}$lead to the same boundary values $\hat{\xi}_{-}=\left.\xi_{-}\right|_{\sigma}$, concluding the proof of the theorem.

Definition 5.9 Given conjugate tunneling maps $\left(v_{+}, v_{-}\right)$, let $d \Delta^{Z}$ be the space of deformations of conjugate tunneling maps, restricted to $\sigma$, ie

$$
\begin{aligned}
d \Delta^{Z}= & \left\{\left(\hat{\xi}_{+}, \hat{\xi}_{-}\right) \in \Gamma\left(\widehat{v}_{+}^{*} T Z \oplus \widehat{v}_{-}^{*} T Z\right) \mid \text { there exist } \xi_{ \pm} \in \Gamma\left(v_{ \pm}^{*} T Z\right), h \in T_{j} \mathcal{J}(S)\right. \\
& \text { with }\left.\xi_{ \pm}\right|_{\sigma}=\widehat{\xi}_{ \pm} \text {satisfying } D_{\left(v_{ \pm}, j\right)}\left(\xi_{ \pm}, h\right)=0, \text { Equations (5.30), (5.31) } \\
& \text { and (5.32), and the vanishing condition on } \lambda \text { along } Q .\}
\end{aligned}
$$

By Definition 3.9, we identify $\sigma=\sigma_{0}=\sigma_{1}$ and therefore we may identify $\widehat{v}_{ \pm}^{*} T Z$ with $\widehat{u}_{ \pm}^{*} T Z$, when $\left(u_{+}, u_{-}\right)$is a folded map with tunneling maps $\left(v_{+}, v_{-}\right)$. Thus we may also view space of deformations of folded diagonal $d \Delta^{Z}$ as a subset of

$$
\Gamma\left(\widehat{u}_{+}^{*} T Z \oplus \hat{u}_{-}^{*} T Z\right) \subset \Gamma\left(\widehat{u}_{+}^{*} T X \oplus \hat{u}_{-}^{*} T X\right) .
$$

Note that if $\left(\hat{\xi}_{+}, \hat{\xi}_{-}\right) \in d \Delta^{Z}$, and $\eta \in T_{\mathrm{Id}} \operatorname{Diff}\left(\Sigma_{1}, \sigma\right)$ is an infinitesimal gauge transformation tangent to the domain fold $\sigma$, then also $\left(\hat{\xi}_{+}+d u_{+}(\eta), \hat{\xi}_{-}+d u_{-}(\eta)\right) \in d \Delta^{Z}$. This defines an action

$$
T_{\mathrm{Id}} \operatorname{Diff}(\Sigma, \sigma) \times d \Delta^{Z} \rightarrow d \Delta^{Z}
$$

It will prove convenient to extend the definition of deformations of folded diagonal to sections of $\widehat{u}_{+}^{*} T X \oplus \widehat{u}_{-}^{*} T X$ in such a way that the action (5.42) extends to all infinitesimal gauge transformations $T_{\mathrm{Id}} \operatorname{Diff}(\Sigma)$ of the map domain, including the ones that move the domain fold. This will greatly simplify taking the quotient by the gauge action later. The following definitions and lemmas facilitate this. 
Definition 5.10 Let $\left(u_{+}, u_{-}\right)$be a folded holomorphic map with conjugate tunneling maps $\left(v_{+}, v_{-}\right)$.

Let $H_{ \pm}=u_{ \pm}^{*} T X$ and set $\hat{H}_{ \pm}=\left.H_{ \pm}\right|_{\sigma}$. We define the subbundles of $\hat{H}_{ \pm}$

$$
F_{ \pm}=\widehat{u}_{ \pm}^{*} F \quad \text { and } \quad E_{ \pm}=\widehat{u}_{ \pm}^{*} E .
$$

Recall the $\left(J_{+}, J_{-}\right)$-linear operators $A^{F}: F_{+} \rightarrow F_{-}$and $A^{E}: E_{+} \rightarrow E_{-}$defined in Definition 5.2. Let

$$
\widetilde{Q}: \Gamma\left(E_{-}\right) \rightarrow \Gamma\left(v_{-}^{*} E\right)
$$

be the operator so that for $\zeta^{K}, \zeta^{L}: \sigma \rightarrow \mathbb{R}$ we have that $\zeta_{1} \partial_{r}+\zeta_{2} R=\widetilde{Q}\left(\zeta^{K} \partial_{r}+\zeta^{L} R\right)$ satisfies

$$
\begin{aligned}
d \zeta_{1} \circ j+d \zeta_{2} & \in \mathcal{H}_{n}(S) \\
\left.\zeta_{1}\right|_{\sigma} & =\zeta^{K} \\
\zeta_{2}(p) & =0 .
\end{aligned}
$$

Define the maps

$$
\begin{array}{ll}
D: \Gamma\left(E_{-}\right) \rightarrow \Gamma\left(E_{-}\right), & D\left(\zeta^{K} \cdot \partial_{r}+\zeta^{L} \cdot R\right)=\zeta^{K} \cdot \partial_{r}-\zeta^{L} \cdot R \\
Q: \Gamma\left(E_{-}\right) \rightarrow \Gamma\left(E_{-}\right), & Q(\zeta)=\left.\widetilde{Q}(\zeta)\right|_{\sigma} \\
C: \Gamma\left(E_{+}\right) \rightarrow \Gamma\left(E_{-}\right), & C=-D \circ Q \circ A^{E} \\
f_{\mathbb{C}}: F_{+} \rightarrow E_{+}, & f_{\mathbb{C}}(\chi)=f(\chi) \otimes \partial_{r}-f(J \chi) \otimes R .
\end{array}
$$

Note that $D$ is $J_{-}$antilinear and $f_{\mathbb{C}}$ is $J_{+}$linear.

Lemma 5.11 $C$ is a pseudodifferential operator of order zero with principal symbol

$$
c(s, \zeta)=-\left(\mathrm{Id}-i J_{-}\right) \pi_{K} A^{E} \zeta .
$$

Proof We claim that $Q$ is a pseudodifferential operator of order zero with principal symbol

$$
q(s, \zeta)=\frac{1}{2}\left(\mathrm{Id}+i J_{-}\right)(\zeta+D \zeta)=\left(\operatorname{Id}+i J_{-}\right) \pi_{K} \zeta .
$$

To see this we note that $Q$ can be written as

$$
Q=P+D \circ(1-P)
$$

where $P$ is the Calderón projector onto the space of Cauchy data of the elliptic operator $\widetilde{Q}$. Then recall that $\sigma$ has the opposite orientation from $\partial S$, so the principal symbol $p$ of $P$ is just the projection $p=\frac{1}{2}\left(\mathrm{Id}+i J_{-}\right)$onto the $J_{-}$antiholomorphic subspace of $E_{-}$. For a detailed discussion of this see Seeley [23] or Booß-Bavnbek 
and Wojciechowski [3] in the case of Cauchy-Riemann operators, and see Nicolaescu [20, Section 6.2] for the family version, interpreting $Q$ as a family of Cauchy-Riemann operators with parameter space $\mathcal{H}_{n}(S)$.

Thus using the principal symbol $q$, we have

$$
\begin{aligned}
c & =-D \circ q \circ A^{E}=-D\left(\mathrm{Id}+i J_{-}\right) \pi_{K} A^{E}=-\left(\mathrm{Id}-i J_{-}\right) D \pi_{K} A^{E} \\
& =-\left(\mathrm{Id}-i J_{-}\right) \pi_{K} A^{E} .
\end{aligned}
$$

Definition 5.12 Let $\left(\Sigma, \sigma, j, u_{+}, u_{-}\right)$be a folded holomorphic map. Using the splitting $\widehat{H}_{ \pm}=F_{ \pm} \oplus E_{ \pm}$we define the linear map on sections

$$
\begin{gathered}
B: \Gamma\left(\hat{H}_{+}\right) \rightarrow \Gamma\left(\hat{H}_{-}\right) \\
B\left(\xi^{F} \oplus \xi^{E}\right)=A^{F}\left(\xi^{F}\right) \oplus A^{E}\left(\xi^{E}-f_{\mathbb{C}}\left(\xi^{F}\right)\right)+C\left((1-a) \xi^{E}-f_{\mathbb{C}}\left(\xi^{F}\right)\right)
\end{gathered}
$$

where $a$ is the gap-function from Equation (3.12).

Lemma 5.13 The deformations of the folded diagonal are given by the graph of the operator $B$ defined in (5.44), restricted to $\hat{u}_{+}^{*} T Z$, ie

$$
d \Delta^{Z}=\operatorname{graph}\left(\left.B\right|_{\widehat{u}_{+}^{*} T Z}\right) .
$$

Proof For $\hat{\xi} \in \Gamma\left(\widehat{u}_{+}^{*} T Z\right)$,

$$
\begin{aligned}
B(\hat{\xi}) & =A^{F}\left[\pi_{F} \hat{\xi}\right]+A^{E}\left[\pi_{E} \hat{\xi}-f_{\mathbb{C}}\left(\pi_{F} \hat{\xi}\right)\right]+C\left[(1-a) \pi_{E} \hat{\xi}-f_{\mathbb{C}}\left(\pi_{F} \hat{\xi}\right)\right] \\
& =A^{F}\left[\pi_{F} \widehat{\xi}\right]-\left[\alpha(\hat{\xi})+f\left(J_{F} \hat{\xi}\right)\right] R-f\left(\pi_{F} \hat{\xi}\right) \partial_{r}+D \circ Q\left[f\left(\pi_{F} \hat{\xi}\right) \partial_{r}\right] \\
& =A^{F}\left[\pi_{F} \hat{\xi}\right]-\left[\alpha(\hat{\xi})+f\left(J_{F} \hat{\xi}\right)\right] R-\pi_{L} Q\left[f\left(\pi_{F} \hat{\xi}\right) \partial_{r}\right] .
\end{aligned}
$$

Then $Q\left(f\left(\pi_{F} \xi\right) \partial_{r}\right)$ is exactly the function $g$ from Equation (5.37) from the proof of Theorem 5.8, so $B(\hat{\xi})$ equals $\left.\xi_{-}\right|_{\sigma}$ from Equation (5.39).

Using $B$ we extend the definition of the folded diagonal to a subset of the space of sections of $\hat{u}_{+}^{*} T X \oplus \hat{u}_{-}^{*} T X$ in the obvious way:

Definition 5.14 The space of extended deformations $d \Delta^{X}$ of the folded diagonal is

$$
d \Delta^{X}=\operatorname{graph}(B)
$$

The following result motivates the definition of $B$ and $d \Delta^{X}$.

Lemma 5.15 The space of extended deformations $d \Delta^{X}$ of the folded diagonal is invariant under the full infinitesimal gauge group of $\Sigma$ (not just the subgroup that preserves $\sigma$ as a set). 
Proof Let $\eta$ be a section of $T_{\sigma} S=T_{\sigma} \Sigma$. Then

$$
\begin{gathered}
f\left(\pi_{F} d u_{+}(\eta)\right)=f\left(\pi_{F} d v_{+}(\eta)\right)=\lambda(\eta), \\
\text { and } \quad(1-a) \pi_{K} d u_{+}(\eta)=(1-a) u_{+}^{*} \alpha(j \eta) \partial_{r}=u_{+}^{*} \alpha(j \eta)+u_{-}^{*} \alpha(j \eta)=\lambda(\eta) \partial_{r} \\
C\left[(1-a) \pi_{E} d u_{+}(\eta)-f_{\mathbb{C}}\left(\pi_{F} d u_{+}(\eta)\right)\right]=C(0)=0 .
\end{gathered}
$$

Then we have

$$
\begin{aligned}
B\left(d u_{+}(\eta)\right) & =A^{F}\left(\pi_{F} d u_{+}(\eta)\right)+A^{E}\left[\pi_{E} d u_{+}(\eta)-f_{\mathbb{C}}\left(\pi_{F} d u_{+}(\eta)\right)\right] \\
& =A^{F}\left(\pi_{F} d v_{+}(\eta)\right)+A^{E}\left[\left(u_{+}^{*} \alpha(j \eta)-\lambda(\eta)\right) \partial_{r}+\left(u_{+}^{*} \alpha(\eta)+\lambda(j \eta)\right) R\right] \\
& =\pi_{F} d v_{-}(\eta)+\left(u_{+}^{*} \alpha(j \eta)-\lambda(\eta)\right) \partial_{r}-\left(u_{+}^{*} \alpha(\eta)+\lambda(j \eta)\right) R \\
& =\pi_{F} d u_{-}(\eta)-u_{-}^{*} \alpha(j \eta) \partial_{r}+u_{-}^{*} \alpha(\eta) R \\
& =\pi_{F} d u_{-}(\eta)+\pi_{E} d u_{-}(\eta) \\
& =d u_{-}(\eta) .
\end{aligned}
$$

\section{Fredholm theory for folded holomorphic maps}

Now we come to the result that justifies the definitions and lemmas pertaining to tunneling maps. We show that they give elliptic boundary values.

Let $\left(u_{+}, u_{-}, j\right)$ be a folded holomorphic map with domain fold $\sigma \subset \Sigma$.

Theorem 6.1 Assume that the map $\left(u_{+}, u_{-}\right)$is transverse to the fold, so $\sigma$ is a manifold. Then the map

$$
R: \Gamma\left(\hat{H}_{+}\right) \oplus \Gamma\left(\hat{H}_{-}\right) \rightarrow \Gamma\left(\hat{H}_{-}\right), \quad \xi_{+} \oplus \xi_{-} \mapsto \xi_{-}-B\left(\xi_{+}\right)
$$

poses elliptic boundary conditions for the folded holomorphic map $\left(u_{+}, u_{-}, j\right)$, ie the principal symbol $r$ of $R$ restricted to the range of the principal symbol $p$ of the Calderón projector $P$ for the complexified Cauchy-Riemann operator $D_{u_{+}} \times D_{u_{-}}$on $H_{+} \otimes \mathbb{C} \times H_{-} \otimes \mathbb{C}$

$$
\left.r\right|_{\text {range }(p)} \rightarrow \hat{H}_{-} \otimes \mathbb{C}
$$

is an isomorphism.

Proof Note that the principal symbol $r$ is given by $r(u, v)=v-b(u)$, where $b$ is the principal symbol of $B$. 
Let $\hat{H}_{ \pm}^{\mathbb{C}}=\hat{H}_{ \pm} \otimes \mathbb{C}$ denote the complexification of $\hat{H}_{ \pm}$and let $\hat{H}_{ \pm}^{\prime}\left(\hat{H}_{ \pm}^{\prime \prime}\right)$ denote the $\left(i, J_{ \pm}\right)$-linear (antilinear) subspace of $\hat{H}_{ \pm}^{\mathbb{C}}$. Recall that $\sigma=\partial \Sigma_{+}^{ \pm}=-\partial \Sigma_{-}$inherits the orientation from $\Sigma_{+}$. Then

$$
\begin{gathered}
p: \hat{H}_{+}^{\mathbb{C}} \oplus \hat{H}_{-}^{\mathbb{C}} \rightarrow \hat{H}_{+}^{\prime} \oplus \hat{H}_{-}^{\prime \prime} \\
p(v, w)=\frac{1}{2}\left(\operatorname{Id}-i J_{+}\right) \oplus \frac{1}{2}\left(\operatorname{Id}+i J_{-}\right)
\end{gathered}
$$

is the projection onto the $\left(i, J_{+} \oplus-J_{-}\right)$linear subspace.

Note that $c=\frac{1}{2}\left(\mathrm{Id}-i J_{-}\right) c$ equals its projection onto $E^{\prime}$. Evaluating $c$ on an element $w \in E_{+}^{\prime}$ gives

$$
\begin{aligned}
c(w) & =-\left(\operatorname{Id}-i J_{-}\right) \pi_{K} A^{E}(w)=-A^{E}\left[\left(\operatorname{Id}-i J_{+}\right) \pi_{K} w\right] \\
& =-A^{E}\left[\pi_{K} w-\pi_{L}\left(i J_{+} w\right)\right]=-A^{E}\left(\pi_{K} w+\pi_{L} w\right) \\
& =-A^{E} w .
\end{aligned}
$$

With the notation from Definition 5.12 and $w=w^{F} \oplus w^{E} \in F_{+}^{\prime} \oplus E_{+}^{\prime}$,

$$
\begin{aligned}
b(w) & =A^{F}\left(w^{F}\right)+A^{E}\left(w^{E}-f_{\mathbb{C}}\left(w^{F}\right)\right)+c\left((1-a) w^{E}-f_{\mathbb{C}}\left(w^{F}\right)\right) \\
& =A^{F}\left(w^{F}\right)+A^{E}\left(w^{E}-f_{\mathbb{C}}\left(w^{F}\right)-(1-a) w^{E}+f_{\mathbb{C}}\left(w^{F}\right)\right) \\
& =A^{F}\left(w^{F}\right)+a A^{E}\left(w^{E}\right) .
\end{aligned}
$$

Now suppose that $(w, z) \in \operatorname{range}(p)=\hat{H}_{+}^{\prime} \oplus \hat{H}_{-}^{\prime \prime}$ and $r(w, z)=0$. Then necessarily $w^{F}=0$ and $w^{E}=0$ as $a>0$, and therefore also $z=0$. We conclude that $r$ is an isomorphism.

Now standard theory shows that the linearized operator is Fredholm. For this next theorem we fix the complex structure on the domain $\Sigma=\Sigma_{0}$, and let $\Lambda_{ \pm}^{0,1}$ be the $j$-antilinear part of $T^{*} \Sigma_{ \pm}$.

Theorem 6.2 For any $s \geq 1$, the operator

$$
\begin{aligned}
D_{B}^{s}: H^{s}\left(\Sigma_{+}, H_{+}\right) \times H^{s}\left(\Sigma_{-}, H_{-}\right) & \rightarrow \\
H^{s-1}\left(\Sigma_{+}, \Lambda_{+}^{0,1} \otimes H_{+}\right) & \times H^{s-1}\left(\Sigma_{-}, \Lambda_{-}^{0,1} \otimes H_{-}\right) \times H^{s-\frac{1}{2}}\left(\partial \Sigma_{-}, \hat{H}_{-}\right) \\
\left(\xi_{+}, \xi_{-}\right) & \mapsto\left(D_{u_{+}} \xi_{+}, D_{u_{-}} \xi_{-}, R\left(\hat{\xi}_{+}, \widehat{\xi}_{-}\right)\right)
\end{aligned}
$$

is Fredholm with real Fredholm index

$$
\operatorname{index}\left(D_{B}^{S}\right)=\mu\left(H_{+}, F_{+}\right)+\mu\left(H_{-}, F_{-}\right)+2 \chi(\Sigma)
$$


where $F_{ \pm}=K \oplus \pi_{F} d u_{ \pm}(T \sigma)$ are totally real subbundles and $\mu$ is the Maslov index. The kernel of $D_{B}^{s}$ is independent of choice of $s \geq 1$ and consists only of smooth solutions.

The Fredholm and smoothness properties is a direct application of the following theorems from Hörmander [15] that we state for the convenience of the reader. The same results can also be deduced from Theorems 19.1 and 20.8 of Booß-Bavnbek and Wojciechowski [3].

Theorem 6.3 [15, Theorem 20.1.2] If the boundary problem (6.45) is elliptic and $s \geq 1$, then (6.46) is a Fredholm operator.

Theorem 6.4 [15, Theorem 20.1.8] If the boundary problem (6.45) is elliptic, then the kernel of the Fredholm operator (6.46) is in $C^{\infty}\left(\Sigma_{+}, H_{+}\right) \times C^{\infty}\left(\Sigma_{-}, H_{-}\right)$, and the range is the orthogonal space of a finite-dimensional subspace of

$$
C^{\infty}\left(\Sigma_{+}, \Lambda_{+}^{0,1} \otimes H_{+}\right) \times C^{\infty}\left(\Sigma_{-}, \Lambda_{-}^{0,1} \otimes H_{-}\right) \times C^{\infty}\left(\partial \Sigma_{-}, \hat{H}_{-}\right) .
$$

Thus the index is independent of $s$. It is also independent of the lower order terms in $D_{u_{ \pm}}$and $R$, and is stable under arbitrary small perturbations of the coefficients.

Proof of Theorem 6.2 The Fredholm properties and smoothness hold by Theorem 6.3 and Theorem 6.4 above. To see the index formula, define the homotopy of boundary conditions

$$
B_{t}\left(\xi^{F} \oplus \xi^{E}\right)=A^{F}\left(\xi^{F}\right)+A^{E}\left(\xi^{E}-f_{\mathbb{C}}\left(\xi^{F}\right)\right)+t \cdot C\left((1-a) \xi^{E}-f_{\mathbb{C}}\left(\xi^{F}\right)\right) .
$$

Following the arguments as in the proof of Theorem 6.1 we see that the symbol $b_{t}$ of $B_{t}$ satisfies on $w=w^{E}+w^{F} \in E_{+}^{\prime} \oplus F_{+}^{\prime}$,

$$
\begin{aligned}
b_{t}(w) & =A^{F}\left(w^{F}\right)+A^{E}\left[\left(w^{E}-f_{\mathbb{C}}\left(w^{F}\right)\right]-t A^{E}\left[(1-a) w^{E}-f_{\mathbb{C}}\left(w^{F}\right)\right]\right. \\
& =A^{F}\left(w^{F}\right)+A^{E}\left[(1+a t-t) w^{E}-(1-t) f_{\mathbb{C}}\left(w^{F}\right)\right] .
\end{aligned}
$$

Thus if $b_{t}(w)=0$, then $w^{F}=0$ and $(1+a t-t) A^{E}\left(w^{E}\right)=0$. Using that $a>0$ we see that $1+a t-t>0$ for $t \in[0,1]$ and we conclude that $w^{E}=0$ for all $t \in[0,1]$. Thus each member in this family gives elliptic boundary conditions.

$B_{0}$ is the $\left(J_{+}, J_{-}\right)$-linear bundle isomorphism with

$$
B_{0}(K)=K, \quad B_{0}\left(\pi_{F} d u_{+}(T \sigma)\right)=\pi_{F} d u_{-}(T \sigma),
$$

so $B_{0}\left(F_{+}\right)=F_{-}$. 
Then $\operatorname{graph}\left(B_{0}\right)$ has the same Maslov index as $\left(F_{+}, F_{-}\right)$, as can be seen by the homotopy of totally real subspaces

$$
\Lambda_{t}=\left\{\left(u+(1-t) J_{+} v, B_{0}\left[\left(1-t-J_{+} t\right)\left((1-t) u+J_{+} v\right)\right]\right) \mid u, v \in F_{+}\right\}
$$

satisfying $\quad \Lambda_{0}=\operatorname{graph}\left(B_{0}\right)$ and $\Lambda_{1}=\left(F_{+}, B_{0}\left(F_{+}\right)\right)=\left(F_{+}, F_{-}\right)$.

In conclusion we have that the index for the boundary value problem given by $B=B_{1}$ is the same as the one for $B_{0}$, which in turn can be computed by $\left(F_{+}, F_{-}\right)$.

To visualize the construction and the results up to here consider the following. As seen in Section 2, the diagonal in $\operatorname{Map}(\sigma, Z) \times \operatorname{Map}(\sigma, Z)$ does not yield elliptic boundary conditions. In the language of Nicolaescu [20] we may say that given holomorphic maps $\left(u_{+}, u_{-}\right)$with $\left.u_{+}\right|_{\sigma}=\left.u_{-}\right|_{\sigma}$, the subspace

$\left\{\left(I\left(\hat{\xi}_{+}\right), \widehat{\xi}_{-}\right) \in L^{2}\left(\sigma, \widehat{u}_{-}^{*} T X\right) \times L^{2}\left(\sigma, \widehat{u}_{-}^{*} T X\right) \mid\right.$ there are $\left.\xi_{ \pm} \in \operatorname{ker}\left(D_{u_{ \pm}}\right),\left.\xi_{ \pm}\right|_{\sigma}=\widehat{\xi}_{ \pm}\right\}$

where $I: L^{2}\left(\sigma, \hat{u}_{+}^{*} T X\right) \rightarrow L^{2}\left(\sigma, \hat{u}_{-}^{*} T X\right)$ is the obvious identification, is not a Fredholm pair. But the folded diagonal gives elliptic boundary conditions, or given a folded holomorphic map $\left(u_{+}, u_{-}\right)$the subspace

$\left\{\left(B\left(\hat{\xi}_{+}\right), \widehat{\xi}_{-}\right) \in L^{2}\left(\sigma, \widehat{u}_{-}^{*} T X\right) \times L^{2}\left(\sigma, \hat{u}_{-}^{*} T X\right) \mid\right.$ there are $\left.\xi_{ \pm} \in \operatorname{ker}\left(D_{u_{ \pm}}\right),\left.\xi_{ \pm}\right|_{\sigma}=\xi_{ \pm}\right\}$

is a Fredholm pair.

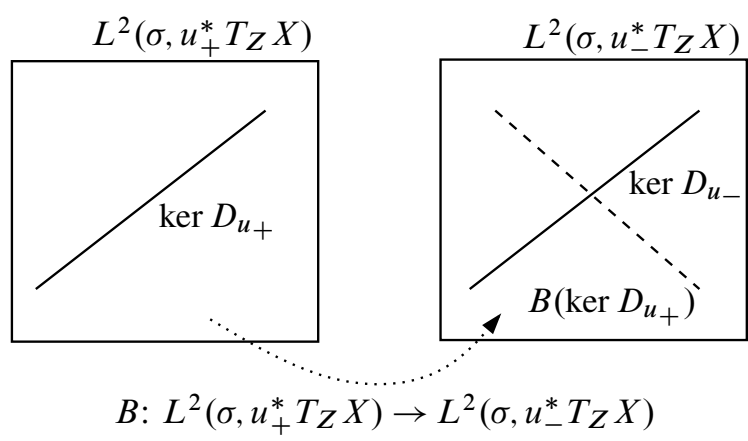

Figure 6: The map $B$ induces a Fredholm pair.

To obtain nice compactifications it is important to first allow for variation in the choice of parametrization of the closed characteristic. This will induce an $S^{1}$-action on the space of solutions, corresponding to the different choices of parametrization. We refer to the quotient by the $S^{1}$-action as the reduced moduli space. 
Remark 6.5 We can generalize Theorem 6.2 by incorporating variations of the folded domain and the choice of parametrization of the closed characteristic. Here are some brief comments on how this can be done.

The first step is allowing variations in $j_{0}$ and $\tau_{0}$, modulo $\operatorname{Diff}^{+}\left(\Sigma_{0}\right)$. Note that $\tau_{0}$ is determined by the map by Equation (3.17). Since the folded diagonal is invariant under the gauge action of $\operatorname{Diff}^{+}\left(\Sigma_{0}\right)$, Theorem 6.2 holds in this case with the index raised by the dimension of Teichmüller space $-3 \chi\left(\Sigma_{0}\right)$.

Next consider variations in $\tau_{1}, j_{1}, \psi$ and $g$, modulo the action of the remaining factors of the gauge group $\operatorname{Diff}^{+}\left(\Sigma_{1}\right) \times \operatorname{Map}\left(\Sigma_{1}, \mathbb{R}\right)$. First, for fixed domain location of the domain fold $\sigma_{1}$, the space of holomorphic diffeomorphisms $\psi^{-1}: \Sigma_{0}^{+} \rightarrow \Sigma_{1}^{+}$sending $\sigma_{0}$ to $\sigma_{1}$ (with $j_{0}$ fixed and $j_{1}$ varying) has dimension $2 \chi\left(\Sigma_{1}^{+}\right)+(1-3) \chi\left(\Sigma_{1}^{+}\right)=0$. Thus variations in $\psi$ and $j_{1}$ on $\Sigma_{1}^{+}$do not change the dimension count.

Finally, note that $g$ is determined by choice of $\tau_{1}$ and $\psi$ by Equation (3.10), and that the deformations of complex structure $j_{1}$ on $S$ are fixed by the tunneling map. Thus the freedom left in choosing $\tau_{1}, j_{1}, \psi, g$ is exactly given by the remaining part $\operatorname{Diff}^{+}\left(\Sigma_{1}\right) \times \operatorname{Map}\left(\Sigma_{1}, \mathbb{R}\right)$ of the gauge group $\mathcal{G}$. Changing the parametrization of the closed characteristic raises the index by 1 . In conclusion, when varying the folded domain and the parametrization of the closed characteristic, and taking the quotient by the gauge group, the index is

$$
\mu\left(u_{+}, K_{+}\right)+\mu\left(u_{-}, K_{-}\right)+(2-3) \chi(\Sigma)+1 .
$$

\subsection{Homological data}

A folded map $\left(u_{+}, u_{-}\right)$together with a pair of conjugate tunneling maps $\left(v_{+}, v_{-}\right)$ gives rise to two relative homology classes

$$
A_{ \pm} \in H_{2}\left(X^{ \pm}, \mathcal{R} ; \mathbb{Z}\right) \quad \text { and } \quad A_{ \pm}=\left(u_{ \pm} \sqcup_{\sigma} v_{ \pm}\right)_{*}\left[\tilde{\Sigma}_{ \pm}\right]
$$

Since the map gluing the tunneling domain $\widehat{S}$ to $\Sigma_{+}\left(\Sigma_{-}\right)$is orientation-preserving (reversing), and the $\omega$-energies of the tunneling maps agree by Definition 4.5 , we obtain the energy identities

$$
\begin{aligned}
E_{\omega}\left(u_{+}\right)+E_{\omega}\left(v_{+}\right) & =\omega \cdot A_{+}=\mathrm{const} \\
E_{\omega}\left(u_{-}\right)-E_{\omega}\left(v_{-}\right) & =\omega \cdot A_{-}=\mathrm{const} \\
E_{\omega}\left(u_{+}\right)+E_{\omega}\left(u_{-}\right) & =\omega \cdot\left(A_{+}+A_{-}\right)=\mathrm{const} .
\end{aligned}
$$

Therefore the space of folded holomorphic maps breaks up into components labeled by the relative homology classes $A_{ \pm}$, and the sum of the $\omega$-energies of the maps $u_{+}$and $u_{-}$is constant in families. 


\section{Examples of folded holomorphic maps}

We give examples of folded holomorphic maps in two special cases.

\subsection{Folded holomorphic maps into folded $E(1)$}

We come back to the example of $E(1)$ from Section 2 and show that Definition 2.1 and Definition 3.10 coincide.

Note that in this case $d \alpha=0$ so Equations (4.22) and (4.23) decouple. After fixing a closed characteristic with parametrizations $x_{\theta_{0}}(\theta)=\left(e^{2 \pi i\left(\theta-\theta_{0}\right)}, z_{0}\right)$ it is straightforward to verify that the folded diagonal is given by the graph of the family of functions

$$
\Phi_{\theta_{0}}: S^{1} \times T^{2} \rightarrow S^{1} \times T^{2}, \quad \Phi_{\theta_{0}}\left(e^{2 \pi i\left(\theta_{0}+\theta\right)}, z\right)=\left(e^{2 \pi i\left(\theta_{0}-\theta\right)}, z\right) .
$$

Note that the space of folded holomorphic maps of degree $d$ carries a free $S^{1}$-action given by the choice of $\theta_{0} \in S^{1}=\mathbb{R} / \frac{1}{2} \mathbb{Z}$ since $\theta_{0}$ and $\Phi_{\theta_{0}}=\Phi_{\theta_{0}+\frac{1}{2}}$. We interpret this as the different choices in gluing the folded symplectic $E(1)$.

This reproduces Definition 2.1.

\subsection{Folded holomorphic rational degree 1 curves in $S^{4}$}

We explicitly characterize the moduli space of folded holomorphic degree 1 rational curves by utilizing the symmetries of the folded symplectic and complex structure on $S^{4}$ defined in Section 2. Essentially these curves come from pseudoholomorphic curves in $\mathbb{P}^{2}$.

Recall that the cylinder over $S^{3}$ ("symplectization") with its standard $\mathbb{R}$-invariant structure is biholomorphic to $\mathbb{C}^{2} \backslash\{0\}$ via

$$
\Phi: \mathbb{R} \times S^{3} \rightarrow \mathbb{C}^{2} \backslash\{0\}, \quad(t, z) \mapsto e^{2 t} z .
$$

For the rest of this section we fix homogeneous coordinates $[x: y: z]$ on $\mathbb{P}^{2}$ and a corresponding embedding $\mathbb{C}^{2} \subset \mathbb{P}^{2},(z, w) \mapsto[x: w: 1]$, whose complement is denoted by $\mathbb{P}_{\infty}^{1}$. Using this we can view finite asymptotic energy pseudoholomorphic maps in $\mathbb{R} \times S^{3}$ as maps in $\mathbb{P}^{2}$. Conversely, pseudoholomorphic maps in $\mathbb{P}^{2}$ that have no components that lie entirely in $\mathbb{P}_{\infty}^{1} \cup\{0\}$ can be viewed as (punctured) pseudoholomorphic maps into $\mathbb{R} \times S^{3}$ by restriction. A straightforward calculation reveals that punctured finite asymptotic energy pseudoholomorphic maps into $\mathbb{R} \times S^{3}$ extend over the punctures to pseudoholomorphic maps into $\mathbb{P}^{2}$, and that conversely 
maps into $\mathbb{R} \times S^{3}$ that are restrictions of pseudoholomorphic maps into $\mathbb{P}^{2}$ have finite asymptotic energy.

Note that

$$
H_{2}\left(B^{4}, \mathcal{R} ; \mathbb{Z}\right)=S^{2} \times \mathbb{Z}
$$

where the isomorphism is given by specifying the closed characteristic and the multiplicity. We fix the closed characteristic parametrized by the family

$$
x_{m}(\theta)=\left(m e^{2 \pi i \theta}, 0\right) \subset S^{3}, \quad m \in S^{1}=\{z \in \mathbb{C}|| z \mid=1\}
$$

and the degree $d=1$.

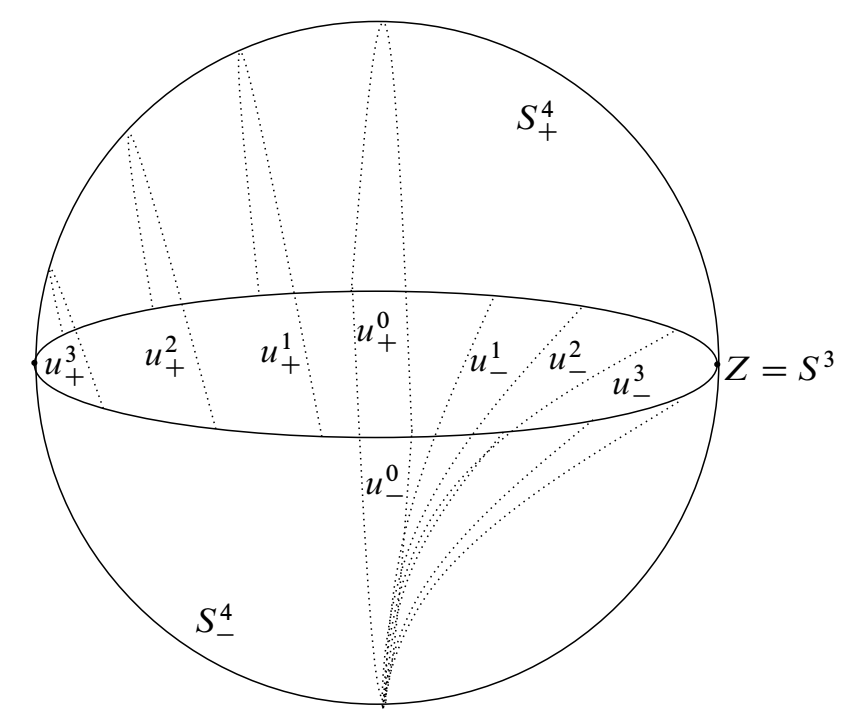

Figure 7: Several members of the moduli space of degree 1 maps into $S^{4}$. The map $\left(u_{-}^{0}, u_{+}^{0}\right)$ corresponds to the case $c=0$. The maps $u_{+}^{n}$ loose energy as they disappear into the fold whereas the maps $u_{-}^{n}$ gain energy.

The open part of the moduli space is parametrized by $c \in \mathbb{D}=\{z \in \mathbb{C}|| z \mid<1\}$ and $m \in S^{1}$. Set

$$
\begin{aligned}
\Sigma_{+} & =\{z \in \widehat{\mathbb{C}}|| z \mid \leq 1\} & \Sigma_{-} & =\{z \in \widehat{\mathbb{C}}|| z \mid \geq 1\} \\
S & =\left\{z \in \widehat{\mathbb{C}}|| z \mid \geq \sqrt{1-|c|^{2}}\right\} & \dot{S} & =S \backslash\{\infty\}
\end{aligned}
$$


where we work in $S^{2}=\widehat{\mathbb{C}}=\mathbb{C} \cup\{\infty\}$. The isomorphism $\psi$ from $\Sigma_{+}$to the set $\Sigma_{1}^{+}=\left\{z \in \mathbb{C}|| z \mid \leq \sqrt{1-|c|^{2}}\right\}$ is given by $z \mapsto \sqrt{1-|c|^{2}} z$. Then set

$$
\begin{array}{ll}
u_{+}(z)=\sigma_{+}\left(\sqrt{1-|c|^{2}} m z, m c\right) & u_{-}(z)=\sigma_{-}\left(\sqrt{1-|c|^{2}} m / z, m c / z^{2}\right) \\
v_{+}(z)=\pi_{S^{3}}(m z, m c) & v_{-}(z)=\pi_{S^{3}}\left(m / z, m c / z^{2}\right) .
\end{array}
$$

We can parametrize this part of the moduli space, modulo reparametrizations of the domain, by keeping track of one of the two intersection points, say $\left(m \sqrt{1-|c|^{2}}, m c\right)$, of $u_{+}$and $u_{-}$, which is in bijective correspondence with $Z \backslash y$, where $y$ is the closed characteristic $y=\{(0, z)|| z \mid=1\} \in \mathcal{R}$.

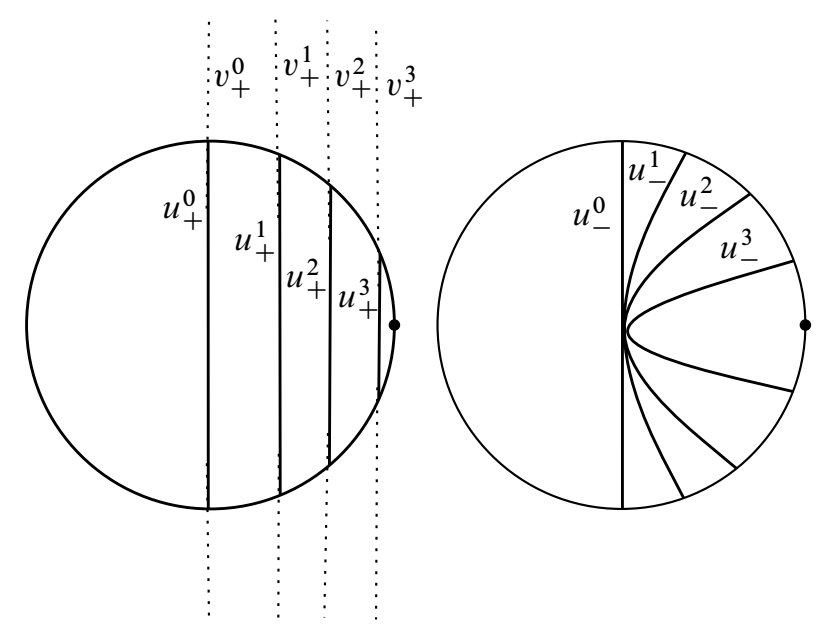

Figure 8: Here we visualize the degree 1 maps as maps into $\mathbb{C}^{2}$. We also suspend the tunneling maps to $(\mathcal{H}-)$ holomorphic maps into $\mathbb{C}^{2}$. The maps $u_{ \pm}$have image in the unit ball, whereas $v_{+}$has image outside the unit ball. $v_{-}$coincides with $u_{-}$.

As the parameter $c$ leaves all compact subsets of $\mathbb{D}$, the domain $\Sigma_{1}$ degenerate so that $\dot{S}=\mathbb{C} \backslash\{0\}$ is a sphere with two punctures, so $v_{ \pm}$form a "bubble" in the fold. The limit maps as $|c| \rightarrow 1 u_{+}(z)=(0, m c) \subset S^{3}$ sink into the fold and become a point map with image on the closed characteristic $y$. All energy is carried by $u_{-}(z)=\left(0, c / z^{2}\right)$, which differ by a reparametrization of the domain for different choices of $|c|=1$. So again we parametrize this portion of the moduli space, modulo reparametrizations of the domain, by the corresponding intersection point $(0, m c) \in y$, noting that any fixed choice of $|c|=1$, while varying $m$, gives the same space of maps.

This shows that the moduli space of degree 1 rational maps has a natural compactification to $S^{3}$. It carries a free $S^{1}$-action given by the choice of parametrization $m$ of the 
closed characteristic. The reduced moduli space, ie the quotient of the moduli space by this action is naturally identified with $S^{2}$, with quotient map the Hopf map.

\subsection{Degree $d$ maps into $S^{4}$}

We briefly indicate how to construct examples of degree $d$ maps from a genus $g$ folded domain $\Sigma$ into $S^{4}$. Start with a degree $d$ curve $w: \Sigma^{2} \rightarrow \mathbb{P}^{2}$ that intersects the $\mathbb{P}^{1}$ at infinity at the closed characteristic $x$. Let $\Sigma_{+}=\{z \in \Sigma \mid\|w(z)\| \leq 1\}$ in the homogeneous coordinates on the complement of the $\mathbb{P}^{1}$ at infinity chosen above. Set $\Sigma_{-}=\Sigma \backslash \Sigma_{+}$and assume for now that $\Sigma_{-}$is simply connected and $\pi_{F} d w \neq 0$ on $\Sigma_{-}$. Then, with $S=\Sigma_{-}$and the puncture corresponding to the preimage of the $\mathbb{P}^{1}$ at infinity we set

$$
u_{+}=\left.\sigma_{+} \circ w\right|_{\Sigma_{+}} \quad \text { and } \quad v_{+}=\left.\pi_{S^{3}} w\right|_{\dot{S}}
$$

To obtain the corresponding maps $v_{-}$and $u_{-}$let $\mu \in \widehat{S} \backslash \dot{S}$ correspond to the direction given by the positive real line in $\mathbb{C}$ and let $f: \dot{S} \rightarrow \mathbb{C}$ be the unique holomorphic function satisfying $x_{m}(\Im(f(p)))=-2 v_{+}(\mu)$, where we use the group structure induced by $x_{m}$, and $d[\Re(f)]=-2 v_{+}^{*} \alpha \circ j$ on $T \sigma$ and set

$$
u_{-}(z)=\sigma_{-}\left(\left.f(z) \cdot w\right|_{\Sigma_{-}}(z)\right) \quad \text { and } \quad v_{-}=\left.\pi_{S^{3}} u_{-}\right|_{\dot{S}}
$$

It is more difficult to construct maps if $\Sigma_{ \pm}$is not simply connected. In this case it will in general not be possible to find a holomorphic function $f$ as above. However, on the subspace of codimension $b_{1}\left(\Sigma_{-}\right)$on which this is possible the above construction still works.

To compute the dimension of the moduli space of degree $d$ maps we compute the index of the linearized operator (6.47) at one of the maps we constructed above that satisfies $\pi_{F} d u_{ \pm} \neq 0$ along the domain fold $\sigma$.

Along each boundary component $\sigma_{i}$ we homotope the boundary conditions $F_{ \pm}(z)$ to $\mathbb{R} \cdot z^{d_{i}^{ \pm}} \times \mathbb{R} \subset u_{ \pm}^{*}(\mathbb{C} \times \mathbb{C})$. Since $w$ was a degree $d$ map into $\mathbb{P}^{2}$ we have that $d=\sum d_{i}^{ \pm}$. Then

$$
\mu\left(H_{ \pm}, F_{ \pm}\right)=\sum \mu\left(\mathbb{C} \times \mathbb{C}, \mathbb{R} \cdot z^{d_{i}^{ \pm}} \times \mathbb{R}\right)=\sum \mu\left(\mathbb{C}, \mathbb{R} \cdot z^{d_{i}^{ \pm}}\right)+\mu(\mathbb{C}, \mathbb{R})=2 d .
$$

Therefore

$$
\text { index }=\mu\left(H_{+}, F_{+}\right)+\mu\left(H_{-}, F_{-}\right)+(2-3) \chi(\Sigma)+1=4 d+2 g-1 .
$$

Again, these spaces carry a free $S^{1}$-action corresponding to the choice $m \in S^{1}$ of parametrization of the closed characteristic. 


\section{Tunneling maps in symplectic manifolds}

Here we want to briefly explain how tunneling maps come up in the usual symplectic setting. From this point of view, tunneling maps appear as tools for studying $J-$ holomorphic curves relative to a codimension 1 hypersurface in a symplectic manifold. We transfer our definitions to this case:

Let $(X, \omega)$ be a symplectic manifold and $f: X \rightarrow \mathbb{R}$ a smooth function with transverse zeros. Then $Z=f^{-1}(0)$ is a smooth hypersurface, separating $X$ into two parts labeled $X_{+}$and $X_{-}$by the sign of $f$ on them. Assume that there exists a $1-$ form $\alpha$ on $Z$ so that $Z$ together with $\alpha$ and $\omega$ admits an $S^{1}$-invariant structure as in Definition 1.6. Choose a compatible almost complex structure $J$ on $X$ that is $S^{1}$-invariant over $Z$.

Fix a folded domain as in Definition 3.1 and let $u_{ \pm}: \Sigma_{ \pm} \rightarrow X_{ \pm}$be $J$-holomorphic with $\tau=u^{*} f$. Assume $\tau$ vanishes transversely and $\sigma=\tau^{-1}(0) \neq \varnothing$, so $\sigma$ is a smooth nonempty compact submanifold of $\Sigma$, separating $\Sigma$.

Now, just like in the folded symplectic case we may look for tunneling maps in $Z$ that connect the image of $\left.u\right|_{\sigma}$ to closed characteristics, ie an $\mathcal{H}$-holomorphic map $v: \dot{S} \rightarrow Z$ with $\left.v\right|_{\sigma}=\left.u\right|_{\sigma}$. One might at first expect that tunneling maps should be pseudoholomorphic instead of $\mathcal{H}$-holomorphic, but note that the index for pseudoholomorphic tunneling maps that are immersions is negative the first Betty number of the domain $-b^{1}(S)$.

As opposed to the folded symplectic case, the complex structures $J_{ \pm}$induced on $T_{Z} X$ coming from $X_{+}$and $X_{-}$agree in the symplectic setting. Thus the argument in the discussion of the sign of $u^{*} \alpha$ in Remark 3.12 has to be modified. Therefore the folded diagonal $\Delta^{Z}$ does not pose Fredholm boundary conditions in the symplectic case as becomes clear in the proof of Theorem 6.2.

To understand this better we take another look at tunneling maps in the folded symplectic setting. Let $\left(v_{+}, v_{-}\right)$be conjugate tunneling maps and consider the suspension $\widetilde{v}_{ \pm}$ of tunneling maps $v_{ \pm}$into $\mathbb{R} \times Z$, where $v_{ \pm}$is a $\mathcal{H}$-holomorphic map with respect to $J_{ \pm}$. Assume for simplicity that $S$ is a punctured disk, so the tunneling maps are actually $J_{ \pm}$-holomorphic. Then the Equation (4.23) of the definition of conjugate tunneling maps can be rewritten in terms of the $\mathbb{R}-$ components $a_{+}$and $a_{-}$of the corresponding tunneling maps. Then

$$
\widetilde{v}_{+}^{*} \alpha \circ j+\widetilde{v}_{-}^{*} \alpha \circ j=d a_{+}-d a_{-}
$$

and using the fact that the $\mathbb{R}$-component is only determined up to a constant, Equation (4.23) says

$$
a_{+}=a_{-} \quad \text { on } \sigma \text {. }
$$


Written in this way the equation carries over verbatim to the (nonfolded) symplectic setting.

We can follow the above transformations backward and obtain the replacement of Equation (4.23) for the symplectic setting:

$$
v_{+}^{*} \alpha \circ j=v_{-}^{*} \alpha \circ j \text { on } T \sigma
$$

for $\mathcal{H}$-holomorphic maps $v_{ \pm}$.

But this, together with the remaining equations in Definition 4.5 implies that $v_{+}=v_{-}$. Thus in the symplectic case, the analogue of the folded diagonal is the actual diagonal

$$
\Delta=\{(\widehat{v}, \widehat{v}) \mid \widehat{v}: \sigma \rightarrow Z\} \subset \operatorname{Map}(\sigma, Z) \times \operatorname{Map}(\sigma, Z) .
$$

Viewing this the other way, the folded diagonal $\Delta^{Z}$ in the folded symplectic setting is analogue of the actual diagonal $\Delta$ in the symplectic setting.

Acknowledgments I thank my adviser Thomas H Parker for his support, guidance and helpful discussions.

\section{References}

[1] C Abbas, K Cieliebak, $\mathbf{H}$ Hofer, The Weinstein conjecture for planar contact structures in dimension three, Comment. Math. Helv. 80 (2005) 771-793 MR2182700

[2] R Baykur, Kähler decomposition of 4-manifolds, Algebr. Geom. Topol. 6 (2006) 1239-1265 MR2253445

[3] B Booß-Bavnbek, K P Wojciechowski, Elliptic boundary problems for Dirac operators, Mathematics: Theory \& Applications, Birkhäuser, Boston (1993) MR1233386

[4] F Bourgeois, Y Eliashberg, H Hofer, K Wysocki, E Zehnder, Compactness results in symplectic field theory, Geom. Topol. 7 (2003) 799-888 MR2026549

[5] A Cannas da Silva, Fold-Forms on Four-Folds, preprint (2002)

[6] A Cannas da Silva, V Guillemin, C Woodward, On the unfolding of folded symplectic structures, Math. Res. Lett. 7 (2000) 35-53 MR1748286

[7] D L Dragnev, Fredholm theory and transversality for noncompact pseudoholomorphic maps in symplectizations, Comm. Pure Appl. Math. 57 (2004) 726-763 MR2038115

[8] G F D Duff, D C Spencer, Harmonic tensors on Riemannian manifolds with boundary, Ann. of Math. (2) 56 (1952) 128-156 MR0048137

[9] Y Eliashberg, A Givental, H Hofer, Introduction to symplectic field theory, Geom. Funct. Anal. (2000) 560-673 MR1826267GAFA 2000 (Tel Aviv, 1999) 
[10] Y Eliashberg, S S Kim, L Polterovich, Geometry of contact transformations and domains: orderability vs. squeezing arXiv:math.SG/0511658

[11] M Gromov, Pseudoholomorphic curves in symplectic manifolds, Invent. Math. 82 (1985) 307-347 MR809718

[12] H Hofer, K Wysocki, E Zehnder, Properties of pseudoholomorphic curves in symplectisations I: Asymptotics, Ann. Inst. H. Poincaré Anal. Non Linéaire 13 (1996) 337-379 MR1395676

[13] H Hofer, K Wysocki, E Zehnder, Properties of pseudoholomorphic curves in symplectizations III: Fredholm theory, from: "Topics in nonlinear analysis", Progr. Nonlinear Differential Equations Appl. 35, Birkhäuser, Basel (1999) 381-475 MR1725579

[14] H Hofer, E Zehnder, Symplectic invariants and Hamiltonian dynamics, Birkhäuser Advanced Texts: Basler Lehrbücher, Birkhäuser Verlag, Basel (1994) MR1306732

[15] L Hörmander, The analysis of linear partial differential operators III, Grundlehren der Mathematischen Wissenschaften 274, Springer, Berlin (1985) MR781536

[16] E-N Ionel, T H Parker, Relative Gromov-Witten invariants, Ann. of Math. (2) 157 (2003) 45-96 MR1954264

[17] E-N Ionel, T H Parker, The symplectic sum formula for Gromov-Witten invariants, Ann. of Math. (2) 159 (2004) 935-1025 MR2113018

[18] R B Lockhart, R C McOwen, Elliptic differential operators on noncompact manifolds, Ann. Scuola Norm. Sup. Pisa Cl. Sci. (4) 12 (1985) 409-447 MR837256

[19] J D McCarthy, J G Wolfson, Symplectic normal connect sum, Topology 33 (1994) 729-764 MR1293308

[20] L I Nicolaescu, Generalized symplectic geometries and the index of families of elliptic problems, Mem. Amer. Math. Soc. 128 (1997) xii+80 MR1388897

[21] L I Nicolaescu, Geometric connections and geometric Dirac operators on contact manifolds, Differential Geom. Appl. 22 (2005) 355-378 MR2166128

[22] M Schwarz, Cohomology operations from $S^{1}$-cobordisms in Floer homology, $\mathrm{PhD}$ thesis, ETH (1995)

[23] R T Seeley, Singular integrals and boundary value problems, Amer. J. Math. 88 (1966) 781-809 MR0209915

[24] C H Taubes, The structure of pseudo-holomorphic subvarieties for a degenerate almost complex structure and symplectic form on $S^{1} \times B^{3}$, Geom. Topol. 2 (1998) 221-332 MR1658028

[25] C H Taubes, A compendium of pseudoholomorphic beasts in $\mathbb{R} \times\left(S^{1} \times S^{2}\right)$, Geom. Topol. 6 (2002) 657-814 MR1943381

[26] C H Taubes, Pseudoholomorphic punctured spheres in $\mathbb{R} \times\left(S^{1} \times S^{2}\right)$ : moduli space parametrizations, Geom. Topol. 10 (2006) 1845-2044 MR2240906 
[27] C H Taubes, Pseudoholomorphic punctured spheres in $\mathbb{R} \times\left(S^{1} \times S^{2}\right)$ : properties and existence, Geom. Topol. 10 (2006) 785-928 MR2240906

Department of Mathematics, University of Notre Dame

Notre Dame, IN 46556-4618

jvonberg@nd.edu

Proposed: Yasha Eliashberg

Seconded: Rob Kirby, Eleny Ionel
Received: 11 January 2006

Revised: 17 November 2006 